\title{
Mice Lacking Brain/Kidney Phosphate-Activated Glutaminase Have Impaired Glutamatergic Synaptic Transmission, Altered Breathing, Disorganized Goal-Directed Behavior and Die Shortly after Birth
}

\author{
Justine Masson, ${ }^{1,8}$ Michèle Darmon, ${ }^{1}$ Agnès Conjard, ${ }^{2}$ Nao Chuhma, ${ }^{6,8}$ Nicole Ropert, ${ }^{3}$ Muriel Thoby-Brisson, ${ }^{4}$ \\ Arthur S. Foutz, ${ }^{4}$ Sandrine Parrot, ${ }^{5}$ Gretchen M. Miller, ${ }^{9,10}$ Renée Jorisch, ${ }^{9,10}$ Jonathan Polan, ${ }^{6,11}$ Michel Hamon, ${ }^{1}$ \\ René Hen, ${ }^{7,8}$ and Stephen Rayport ${ }^{6,8,9}$ \\ ${ }^{1}$ Unité Mixte de Recherche U677, NeuroPsychoPharmacologie, Faculté de Médecine Pitié-Salpêtrière, 75634 Paris Cedex 13, France, ${ }^{2}$ Institut National de la \\ Santé et de la Recherche Médicale U499, Physiopathologie Métabolique et Rénale, 69372 Lyon Cedex 08, France, ${ }^{3}$ Institut National de la Santé et de la \\ Recherche Médicale U603, Centre National de la Recherche Scientifique Formation de Recherche en évolution 2500, Neurophysiologie Nouvelles \\ Microscopies, 75270 Paris Cedex 06, France, ${ }^{4}$ Centre National de la Recherche Scientifique Unité Propre de Recherche 2216, Neurobiologie Génétique et \\ Intégrative, 91198 Gif sur Yvette, France, ${ }^{5}$ Biochimie Fonctionnelle et Neuropharmacologie, Plate-forme de Physiologie, Université Claude Bernard, 69372 \\ Lyon Cedex 08, France, ${ }^{6}$ Department of Psychiatry, ${ }^{7}$ Department of Pharmacology, and ${ }^{8}$ Center for Neurobiology and Behavior, Columbia University, New \\ York, New York 10032, and Departments of ${ }^{9}$ Neuroscience, ${ }^{10}$ Analytical Psychopharmacology, and ${ }^{11}$ Developmental Psychobiology, New York State \\ Psychiatric Institute, New York, New York 10032
}

\begin{abstract}
Neurotransmitter glutamate has been thought to derive mainly from glutamine via the action of glutaminase type 1 (GLS1). To address the importance of this pathway in glutamatergic transmission, we knocked out GLS1 in mice. The insertion of a STOP cassette by homologous recombination produced a null allele that blocked transcription, encoded no immunoreactive protein, and abolished GLS1 enzymatic activity. Null mutants were slightly smaller, were deficient in goal-directed behavior, hypoventilated, and died in the first postnatal day. No gross or microscopic defects were detected in peripheral organs or in the CNS. In cultured neurons from the null mutants, miniature EPSC amplitude and duration were normal; however, the amplitude of evoked EPSCs decayed more rapidly with sustained $10 \mathrm{~Hz}$ stimulation, consistent with an observed reduction in depolarization-evoked glutamate release. Because of this activitydependent impairment in glutamatergic transmission, we surmised that respiratory networks, which require temporal summation of synaptic input, would be particularly affected. We found that the amplitude of inspirations was decreased in vivo, chemosensitivity to $\mathrm{CO}_{2}$ was severely altered, and the frequency of pacemaker activity recorded in the respiratory generator in the pre-Bötzinger complex, a glutamatergic brainstem network that can be isolated in vitro, was increased. Our results show that although alternate pathways to GLS1 glutamate synthesis support baseline glutamatergic transmission, the GLS1 pathway is essential for maintaining the function of active synapses, and thus the mutation is associated with impaired respiratory function, abnormal goal-directed behavior, and neonatal demise.
\end{abstract}

Key words: respiration; neonatal behavior; glutamate release; lethal phenotype; phosphate-activated glutaminase; GLS1; knock-out mice; glutamatergic neurons; synaptic transmission

\section{Introduction}

Glutamate is the principal excitatory neurotransmitter in the CNS. At birth, the first behavior essential to survival is respira-

Received 0ct. 5, 2005; revised March 22, 2006; accepted March 22, 2006.

This work was supported by grants from the National Institute on Drug Abuse (DA00356 and DA14055 to S.R.), the Institut National de la Santé et de la Recherche Médicale (M.H.), National Alliance for Research on Schizophrenia and Depression Independent Investigator (S.R.) and Young Investigator (J.M.) awards, the Centre National de la Recherche Scientifique (J.M., M.T.-B., A.S.F.), the Frontier Fund of the Columbia Department of Psychiatry (J.M.), and the Philippe Foundation (J.M.). We thank Joao Braz for technical assistance on ES cell culturing; Drs. Bernard Ferrier, Mireille Martin, and Gabriel Baverel for helpful recommendations for GLS1 activity determinations and fruitful discussion on glutamine metabolism; Dr. Luc Denoroy for expert advice on capillary electrophoresis with laserinduced fluorescence detection and Valérie Sauvinet for technical assistance; Monica Mendelsohn for blastocyst injections; John Strumbos and SooYeon Lee for preliminary studies; Dr. Norman Curthoys for the generous gift of the GLS1 CDNA; Dr. Bjørg Roberg for the kind gift of GLS1 antisera; Dr. William Dauer for many helpful discussions and for the flox-STOP cassette; Dr. Etienne Sibille for judicious advice on RT-PCR; Biodoxis for histological analyses; and Dr. Jean Champagnat for helpful comments on this manuscript. tion, and this is mediated by brainstem glutamatergic circuits. Later on in development and continuing into adulthood, glutamatergic synaptic transmission is involved in every CNS circuit. Despite extensive investigations, the source of neurotransmitter glutamate has not been resolved. Because de novo synthesis from $\alpha$-ketoglutarate is minimal in neurons (Peng et al., 1993; Hertz et al., 1999), metabolic studies have suggested that most neurotransmitter glutamate is recycled via the glutamine-glutamate

Correspondence should be addressed to either of the following: Dr. Justine Masson, Unité Mixte de Recherche U677 Institut National de la Santé et de la Recherche Médicale/Université Pierre et Marie Curie, Faculté de Médecine Pitié-Salpêtrière, 91 Bd de l'Hôpital, 75634 Paris Cedex 13, France, E-mail: jumasson@ext.jussieu.fr; or Dr. Stephen Rayport, Psychiatry/Neuroscience, Columbia University, 1051 Riverside Drive, NYSPI Unit 62, New York, NY 10032, E-mail: sgr1@columbia.edu.

D01:10.1523/JNEUROSCI.4241-05.2006

Copyright $\odot 2006$ Society for Neuroscience $\quad 0270-6474 / 06 / 264660-12 \$ 15.00 / 0$ 
neuron-astrocyte shuttle, involving brain/kidney phosphateactivated glutaminase type 1 (GLS1; enzyme commission number 3.5.1.2). A review of studies ranging from physiology to biochemistry and magnetic resonance spectroscopy concluded that the majority $(70 \%)$ of neurotransmitter glutamate is recycled by GLS1, with the remainder reflecting de novo synthesis (Hertz, 2004).

The predominant means of inactivation of released glutamate is by rapid uptake into glia via transporters (Danbolt et al., 1998). Glutamine synthetase, which is expressed exclusively in glia rapidly converts the glutamate to glutamine. Glutamine is then released from astrocytes via System $\mathrm{N}$ transporters and taken up by neurons via System A transporters (Varoqui et al., 2000; Chaudhry et al., 2002). This provides a major flux of glutamine into neurons, which convert glutamine into glutamate via the action of GLS1. The critical role of GLS1 has been supported further by pharmacological experiments in which a near complete depletion of neuronal glutamate was observed after inhibition of glutamine synthesis (Ottersen et al., 1992). Furthermore, direct inhibition of GLS1 by the suicide inhibitor 6-diazo-5-oxoL-norleucine decreased the release of glutamate from cerebrocortical synaptosomes (Bradford et al., 1989), glutamate immunoreactivity in neurons (Conti and Minelli, 1994; Sulzer et al., 1998), and glutamate-dependent secretion of gonadotropinreleasing hormone from hypothalamic explants (Bourguignon et al., 1995).

GLS1 was cloned in 1988 (Banner et al., 1988) and shown to be expressed with high baseline activity in the brain (Beitz and Ecklund, 1988; Farb et al., 1992; Chatziioannou et al., 2003) but also in kidney, intestinal endothelium, fetal liver, lymphocytes, adipocytes, and tumor cells. GLS1 was (other than glutamate itself) the only known marker for the glutamatergic status of neurons (Kaneko and Mizuno, 1988) until recently, when three families of vesicular glutamate transporters were identified with strikingly different distributions in subpopulations of glutamatergic neurons (Bellocchio et al., 2000; Bai et al., 2001; Gras et al., 2002).

Here, we asked how important GLS1 is for glutamatergic synaptic function by knocking out the GLS1 gene in mice. We inserted a STOP cassette ahead of the initiating ATG codon in the GLS1 gene on mouse chromosome 1. GLS1 null mice do not feed properly, show a respiratory deficit, and die shortly after birth. Glutamate release from GLS1 null neurons is impaired, presumably accounting for the lethal phenotype, and revealing the relative importance of the pathway as the source of neurotransmitter glutamate.

\section{Materials and Methods}

Animals. Procedures involving mice and their care were conducted in conformity with the institutional guidelines of the National Institutes of Health Guide for the Care and Use of Laboratory Animals and were performed under protocols approved by the Institutional Animal Care and Use Committees of Columbia University and New York State Psychiatric Institute, and in compliance with Council directive \#87-848, October 19, 1987, Ministère de l'Agriculture et de la Forêt, Service Vétérinaire de la Santé et de la Protection Animale, permissions \#75-974 (M.D.), \#75805 (J.M.), and \# 75-116 (M.H.).

Generation of the GLS1-/- mice. The GLS1 gene was isolated in several Bac clones from a $129 \mathrm{SvEv} / \mathrm{J}$ mouse library (Genome Systems, St. Louis, MO) by using the quasi-complete rat GLS1 cDNA (gift from Dr. Norman P. Curthoys, Colorado State University, Ft. Collins, CO) as a probe (a DraIII-HindIII fragment of $1734 \mathrm{~kb}$ ). A SphI-HindIII fragment containing the first exon of GLS1 gene was cloned in pGEM-3Z $(+)$. The PGKneo gene (in reverse, $\mathrm{Neo}^{\mathrm{r}}$ ) followed by a Stop sequence (Lakso et al., 1992), flanked by loxP sites, was inserted into a new Xho site (created by site-directed mutagenesis) located just before the initiating codon of the GLS1 gene (Fig. 1). W9.5 embryonic stem (ES) cells were electroporated (Bio-Rad Gene Pulser; $800 \mathrm{~V}$ and $3 \mu \mathrm{F}$; Bio-Rad, Hercules, CA) with 30 $\mu \mathrm{g}$ of the targeting construct. These embryonic stem cells were then plated onto mitomycin-treated mouse embryonic fibroblasts for 1 week in the presence of G418 (150 $\mathrm{g} / \mathrm{ml}$ active substance). The G418-resistant clones were screened by Southern blot analysis with a HindIII digest and a ${ }^{32} \mathrm{P}$-labeled outside probe (probe A: 336 bp fragment isolated by PCR from the AvaII-SphI fragment in 5' of the targeting construct). Clones showing homologous recombination were screened further with a Neo probe to ensure that the recombination event was unique. The selected clones were then injected into C57BL6/J blastocysts. These blastocysts were reimplanted in $\mathrm{B} 6 \mathrm{CBAF} 1 / \mathrm{J}$ foster mothers to give chimeras, which were mated to $\mathrm{C} 57 \mathrm{BL} / 6$ mice to produce heterozygotes. The GLS1 mutant colony was kept on a mixed background $129 \mathrm{SvEv} / \mathrm{J}-\mathrm{C}$ C57BL6/J. A three-primer PCR strategy [two primers in the wild-type (WT) locus flanking the mutant cassette and one in the mutant cassette] was used for genotyping. Primers used were as follows: GLS1 forward (5'-CAC ACC CCG TCC CGG ACT TTT TC-3'); GLS1 reverse (5'-TTA AGA GCA GCT CCC GTA GCA TC-3'); and neo reverse (5' -CCA GCT CAT TCC TCC CAC TC-3'). GLS1 forward plus GLS1 reverse amplified a 156 bp band from the $5^{\prime}$ region of exon 1 from the wild-type allele, and GLS1 forward plus neo reverse amplified a $347 \mathrm{bp}$ band from the mutant allele.

Quantitative reverse transcription-PCR. RNA was isolated using TRIZOL (Invitrogen, Gaithersburg, MD) following the protocol of the manufacturer, and quantities were standardized by UV spectrofluorometry. Small PCR products ( $100-200 \mathrm{bp})$ were amplified in quadruplicate on an Opticon real-time PCR machine (MJ Research, Waltham, MA) using universal PCR conditions $\left(65^{\circ} \mathrm{C}\right.$ to $59^{\circ} \mathrm{C}$ touch-down, followed by 35 cycles; 15 at $95^{\circ} \mathrm{C}, 10$ at $59^{\circ} \mathrm{C}$, and 10 at $72^{\circ} \mathrm{C}$ ), as described previously (Galfalvy et al., 2003). Amplification of $150 \mathrm{pg}$ of cDNA was done in $20 \mu \mathrm{l}$ reactions, with $0.3 \times$ Sybr-green, $3 \mathrm{mM} \mathrm{MgCl}_{2}, 200 \mu \mathrm{M}$ dNTPs, $200 \mu \mathrm{M}$ primers, $0.5 \mathrm{U}$ Platinum TaqDNA polymerase (Invitrogen). Primers chosen (forward 5' -GCA CTA CAC TTT GGA CAC CA-3' and reverse 5'-TAG CAA CCC GTC GAG ATT-3') were directed to sequences near the $3^{\prime}$ end of the GLS1 gene, and free of primer-dimers or other nonspecific signal after the 35 cycle run. Results were calculated as relative intensity compared with actin (expression level 1000) and then normalized to the average of the wild-type adult brain samples.

Western blot analysis. Adult cortex $(\mathrm{Cx})$ or heart $(\mathrm{He})$ tissue and total newborn brain were homogenized in Laemmli buffer and sonicated. Two micrograms of total protein from adult mice or $10 \mu \mathrm{g}$ total protein from embryonic mice were separated by $10 \%$ SDS-PAGE and blotted onto nitrocellulose (Bio-Rad). A crude rabbit antiserum raised against rat GLS1 was used at 1:5000 dilution (gift from Dr. Bjørg Roberg, University of Oslo, Norway). Blot was incubated with peroxidase-linked goat antirabbit antibodies (Amersham Biosciences, Piscataway, NJ) at a 1:1000 dilution. Immunoreactive bands were visualized by chemiluminescence following the instructions of the manufacturer (ECL; Amersham Biosciences)

Enzymatic activity. Tissue preparation and measurement of GLS1 activity were performed according to established procedures (Curthoys and Lowry, 1973; Conjard et al., 2002). Neonatal tissues were removed, immediately frozen in liquid nitrogen, and kept at $-80^{\circ} \mathrm{C}$ until analyzed. Homogenates were prepared at $0^{\circ} \mathrm{C}$ in $20 \mathrm{~mm}$ phosphate buffer, $\mathrm{pH}$ 7.4, containing $0.5 \mathrm{~mm}$ EDTA, $5 \mathrm{~mm}$ 2-mercaptoethanol, 25\% (v/v) glycerol, and $0.02 \%(\mathrm{w} / \mathrm{v}) \mathrm{BSA}$ and then warmed to $37^{\circ} \mathrm{C}$ for the assay in a spectrofluorimeter microplate reader.

Postnatal day 0 pup behavioral observations. The procedure, test chamber (see Fig. $3 A$ ), and scoring for the observation of maternally directed orienting behaviors (MDOBs) in neonatal pups followed methodology described previously (Polan and Eljuga, 2002). Just before testing, the dam was administered general anesthesia (ketamine/xylazine) to keep her motionless (except for respirations), allowing the pups to respond to passive maternal cues without active assistance. Anesthesia was adjusted so that the dams did not respond to ambient visual, auditory, or tactile stimulation and was flaccid when held. Postnatal day 0 (P0) pups were held in a nest-temperature, humidified incubator and then individually transferred to a nest temperature-maintained test chamber for $4 \mathrm{~min}$ 
without the dam (the control condition) and then for 4 min with the dam (the test condition) and videoed. Videotapes were transferred onto the hard drive of a personal computer using Dazzle Digital Video Creator (Pinnacle Systems, Mountain View, CA) and then to The Observer Video-Pro software (Noldus Information Technology, Wageningen, The Netherlands) for analysis by an observer blind to genotype.

Time spent in three different orientations (prone, side, and supine) was calculated from the record of orientation changes and expressed as a percentage of total time observed. Individual head, torso, and limb movements were counted. Locomotion counts included all behaviors that resulted in movement across the test platform. Barking was recorded with the aid of a microphone. Mouth/lick counts included any mouth opening or closing and any tongue licking.

Histology. Embryos removed from mother uterus or P0 newborn mice were fixed in $10 \%$ formaldehyde for histopathological analysis (Biodoxis, France). Transverse sections were obtained through the skull (forebrain, midbrain, and hindbrain) and from the thorax, abdomen, and pelvis. Trimmed pieces were processed through paraffin and sectioned at $5 \mu \mathrm{m}$. Sections were stained with hematoxylin, eosin, and safran and examined at the light microscopic level. For analysis of brain structure, tissues from newborn mice were fixed in $4 \%$ paraformaldehyde-PBS, frozen sections made at $10 \mu \mathrm{m}$, and Nissl stained.

In vivo measurement of ventilation. Respiratory activity was measured using a barometric method (Jacquin et al., 1996). A plethysmograph recording chamber $(20 \mathrm{ml})$ equipped with a temperature sensor was connected to a reference chamber. The pressure difference between the recording chamber and reference chamber was measured using a differential pressure transducer (DP-103-12; Validyne, Northridge, CA) connected to a sine wave carrier demodulator (Validyne CD15). The spirogram was stored on a personal computer. Calibrations were made during each data collection period by injecting $5 \mu$ l of air into the recording chamber with a syringe. Each animal was placed in the chamber, which was maintained at $28.0-29.5^{\circ} \mathrm{C}$ and hermetically sealed during the $2 \mathrm{~min}$ recording session. Oral temperature was measured before and after each recording by means of a small flexible thermocouple probe.

We analyzed periods of quiet breathing, after excluding movement artifacts and periods of polypnea (fast, high amplitude breathing pattern). Periods of apneas ( $T_{\mathrm{E}}$ exceeding $1 \mathrm{~s}$ ) were analyzed separately. A computer-assisted method (ACQUIS1 software; BioLogic, Claix, France) was used to measure the duration of inspiration $\left(T_{\mathrm{I}}\right)$ and expiration $\left(T_{\mathrm{E}}\right)$, breathing frequency $\left(f_{\mathrm{R}}\right)$, tidal volume $\left(V_{\mathrm{T}}\right)$, and ventilation $\left(V_{\mathrm{E}}\right)$. Neonates were tested in random order. Respiration was always measured first with animals breathing room air (control), and then the chamber was flushed with a mixture of $6 \% \mathrm{CO}_{2} / 21 \% \mathrm{O}_{2} /$ balance $\mathrm{N}_{2}$ for $1 \mathrm{~min}$ and additional measurements made (hypercapneic challenge).

Primary cell culture. Preparation of primary cell cultures followed previously described methods (Sulzer et al., 1998; Jolimay et al., 2000). The cortices of mouse embryos were dissected at embryonic day 18 (E18). Cells were trypsinized, dissociated, and plated on poly-L-lysine-coated 12-mm-diameter coverslips in four-well culture dishes at a density of $50,000-100,000$ cells per well in complete Neurobasal medium with B27 supplement (Invitrogen, Carlsbad, CA), 1 mm L-glutamine, penicillin G $(10 \mathrm{U} / \mathrm{ml})$, and streptomycin $(10 \mathrm{mg} / \mathrm{ml})$.

Immunofluorescence. Immunofluorescence was performed 6-21 d after plating as described previously (Jolimay et al., 2000). Briefly, coverslips were washed quickly in PBS+ (PBS containing $0.1 \mathrm{mM} \mathrm{CaCl}_{2}$ and $0.1 \mathrm{mM} \mathrm{MgCl}_{2}$ ) and then fixed with $4 \%$ paraformaldehyde for $10 \mathrm{~min}$. After three washes with PBS + , cells were permeabilized with $0.5 \%$ Triton X-100 and treated with $0.1 \%$ Triton X-100/10\% donkey serum (Interchim, Montluçon, France) in PBS + (antibody buffer) to block nonspecific labeling. Incubation with primary antibodies was performed in the antibody buffer overnight at $4^{\circ} \mathrm{C}$. After three washes of $10 \mathrm{~min}$ with PBS+, incubation with the secondary antibodies proceeded for $1 \mathrm{~h}$ at room temperature. For GLS1 immunolabeling, crude rabbit GLS1 antibody (gift from Dr. Bjørg Roberg) was diluted at 1:5000 and then visualized with Alexa 488-conjugated donkey anti-rabbit IgG (1:800 dilution; Interchim). For mitochondrial labeling, anti-human mitochondria mouse $\operatorname{IgG}_{1}$ monoclonal antibody (1:100; Interchim) was visualized with a biotin-HRP complex reaction [anti mouse IgG, biotinylated whole
A
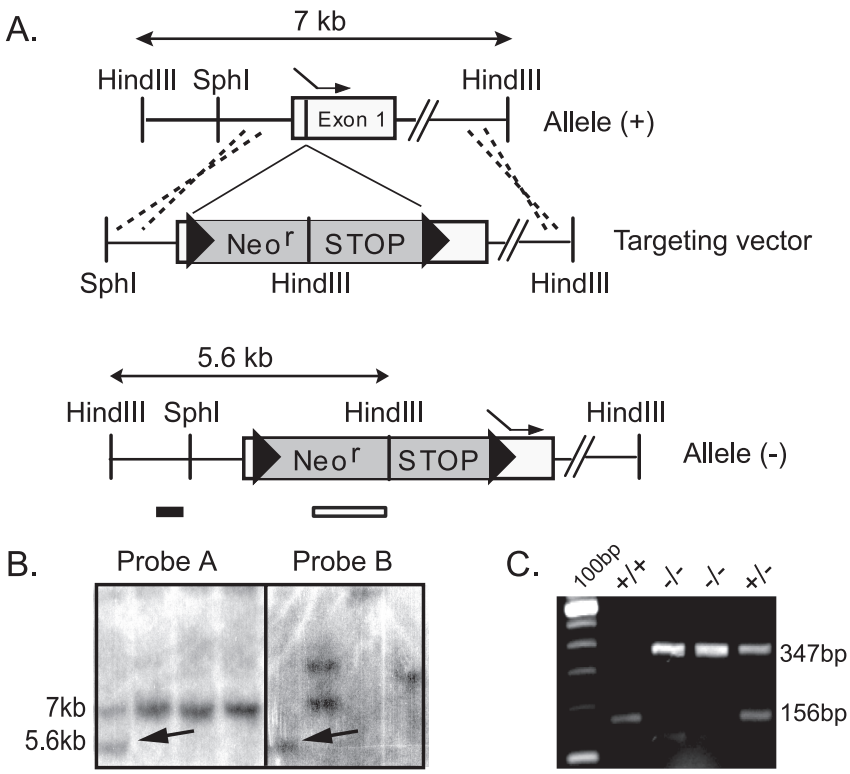

\begin{tabular}{|c|lr|c|c|c|}
\hline \multirow{2}{*}{ Breeding } & \multicolumn{2}{|c|}{ Age (n) } & \multicolumn{3}{|c|}{ Genotype (\%) } \\
\cline { 3 - 6 } & \multicolumn{2}{|c|}{$+/+$} & $+/-$ & $-/-$ \\
\hline$+/-X+/+$ & Adult (148) & 47 & 53 & \\
\hline$+/-X+/-$ & Embryo (154) & 26 & 43 & 31 \\
\cline { 2 - 6 } & Newborn (126) & 28 & 43 & 29 \\
\cline { 2 - 6 } & Adult (132) & 39 & 61 & 0 \\
\hline
\end{tabular}

Figure 1. Targeted disruption of the mouse GLS1. A, Schematic representation of the GLS1 genomic DNA [allele $(+)$ ] and disrupted gene [allele $(-)$ ]. A targeting vector was constructed by introducing a cassette (gray box) containing a neomycin resistant (Neo ${ }^{r}$ ) and a STOP sequence, flanked by two loxP sites (triangles), upstream of the ATG initiating codon (dashed arrow) in the first exon (white box). B, Southern blot analysis of ES cell clones. After digestion of ES cell DNA by Hindlll, probe A (GLS1 probe) hybridized to a $5.6 \mathrm{~kb}$ band for the mutant allele (arrow) and a $7.0 \mathrm{~kb}$ band for the wild-type allele ( + ); probe B (neo probe) hybridized when homologous recombination occurred to a $5.6 \mathrm{~kb}$ band for the mutant allele (arrow). C, PCR GLS1 genotyping. Mice were genotyped using a three-primer PCR strategy (two primers in the wildtype sequence, one in the mutant cassette). The wild-type allele yielded a $156 \mathrm{bp}$ band, and the targeted allele yielded a 347 bp band. D, Genotypic distribution of offspring of GLS1 mutants. Embryos were genotyped at E17-E18, newborns were genotyped at birth, and adults were genotyped at $1-3$ weeks of age ( $n=$ number of animals). GLS1- $/-$ pups died at PO.

antibody from goat (1:100 dilution; Amersham Biosciences), Alexa 555conjugated streptavidin (1:800; Interchim)]. Coverslips were mounted with Vectashield (Vector Laboratories, Burlingame, CA) for fluorescence with 4'-6-diamidino-2-phenylindole (DAPI) (Interchim). Immunofluorescent images were acquired at low magnification $(20 \times$ oil immersion lens) with a Leica (Nussloch, Germany) fluorescence microscope with fluorescein or UV filters, and higher magnification images were acquired with a Leica TCS-400 laser-scanning confocal microscope (100× oil immersion lens). Contrast and brightness were chosen to ensure that all pixels were within the linear range. Confocal images were generated from eightfold line averages.

Spontaneous EPSCs and IPSCs in cortical neurons. For electrophysiological recording, cortical neurons growing on coverslips [8-17 $\mathrm{d}$ in vitro (DIV)] were placed in a recording chamber and perfused at $2 \mathrm{ml} / \mathrm{min}$ with extracellular physiological solution at room temperature $\left(24-26^{\circ} \mathrm{C}\right)$. The neurons were visualized using an upright fixed-stage microscope (Zeiss Axioskop FS; Zeiss, Thornwood, NY) with Nomarski optics. Whole-cell voltage-clamp recordings were made using an Axopatch 200B amplifier (Molecular Devices, Foster City, CA). Recording pipettes were pulled from borosilicate glass tube (1.2 $\mathrm{mm}$ external diam- 
eter) and coated with beeswax. The tip resistance of the recording pipettes was 3-4 $\mathrm{M} \Omega$. The series resistance was estimated by applying voltage steps of $5 \mathrm{mV}$ at the beginning and the end of each recording session. Data were filtered at $5 \mathrm{kHz}$, digitized at $10 \mathrm{kHz}$, and stored directly to disk using a Labmaster TL-1 DMA acquisition board (Molecular Devices). Data were analyzed using Acquis1 software (Biologic). Spontaneous synaptic currents were automatically detected using a threshold-crossing derivative with parameters set for each cell and held constant for each recording session (Perrais and Ropert, 1999). Peak amplitude and $10-90 \%$ rise times were measured. The decay phase of the EPSCs was fitted with a single exponential function.

The extracellular solution contained the following (in mM): $130 \mathrm{NaCl}$, $1.9 \mathrm{KCl}, 1.2 \mathrm{KH}_{2} \mathrm{PO}_{4}, 1.3 \mathrm{MgSO}_{4}, 2.5 \mathrm{CaCl}_{2}, 10$ glucose, $26 \mathrm{NaHCO}_{3}, 310$ mOsm, pH 7.4 (adjusted with $\mathrm{NaOH}$ ). Two pipette solutions were used that contained the following (in mM): (1) 110 CsGluconate, $10 \mathrm{CsCl}, 4$ K-ATP, $0.4 \mathrm{Na}$-GTP, $2 \mathrm{MgCl}_{2}$, 10 HEPES, 10 BAPTA, pH 7.3 (adjusted with $\mathrm{CsOH}$ ), $273 \mathrm{mOsm}$; (2) 130 KGluconate, $5 \mathrm{NaCl}, 2 \mathrm{MgCl}_{2}$, 4 ATP$\mathrm{K}_{2}, 0.5 \mathrm{GTP}-\mathrm{Na}$, 10 HEPES, 0.5 EGTA. Internal solution 1 was used when the holding potential was shifted from negative (near rest at $-70 \mathrm{mV}$ ) to positive values (near $+40 \mathrm{mV}$ ) to reduce the background noise resulting from $\mathrm{K}^{+}$channel activation at positive holding potentials; the second intracellular solution was used when the cells were maintained near rest for miniature EPSC (mEPSC) recordings, which were done under $\mathrm{GABA}_{\mathrm{A}}$ blockade. The following drugs were applied by perfusion: D-2amino-5-phosphonopentanoic acid (D-AP-5; $50 \mu \mathrm{M}$; Tocris Cookson, Ballwin, MO); 6-cyano-7-nitroquinoxaline-2,3-dione (CNQX; $10 \mu \mathrm{M}$; Tocris Cookson); bicuculline hydrochloride (BIC; $10 \mu \mathrm{M}$; Sigma, St. Louis, MO); 2-(3-carboxypropyl)-3-amino-6-(4-methoxyphenyl)pyridazinium bromide (SR95531; $10 \mu \mathrm{M}$; Tocris Cookson); tetrodotoxin (TTX; $1 \mu \mathrm{M}$; Sigma).

In vitro release of glutamate and GABA. Endogenous amino acid release experiments were performed following methods described previously (Pin et al., 1986). Briefly, neuronal cultures at 14-15 DIV were washed twice with $0.5 \mathrm{ml}$ of basal solution (in mM: 20 HEPES, $128 \mathrm{NaCl}$, $5 \mathrm{KCl}, 1.2 \mathrm{MgSO}_{4}, 1.1 \mathrm{CaCl}_{2}, 10$ glucose) prewarmed to $37^{\circ} \mathrm{C}$. Cultures were then incubated in $0.3 \mathrm{ml}$ of basal solution, and the experiment proceeded at $37^{\circ} \mathrm{C}$. For evoked release, cultures were exposed to a stimulatory solution (in mM: 20 HEPES, $77 \mathrm{NaCl}, 56 \mathrm{KCl}, 1.2 \mathrm{MgSO}_{4}, 1.1$ $\mathrm{CaCl}_{2}, 10$ glucose). At 1 min intervals, the bathing solution was removed and replaced with a fresh $0.3 \mathrm{ml}$ of solution. Cultures were tested following a sequence of basal-evoked1-basal-evoked2-basal, changing conditions after three incubations (at $3 \mathrm{~min}$ intervals). Control experiments were performed in the absence of $\mathrm{Ca}^{2+}$ with all solutions containing 1 mM EGTA. Fractions were collected in tubes and then stored at $-80^{\circ} \mathrm{C}$ before analysis of amino acid content. Endogenous GABA and glutamate concentrations in each sample were determined by capillary electrophoresis with laser-induced fluorescence detection. On the day of the analysis, $30 \mu \mathrm{l}$ of sample and $30 \mu \mathrm{l}$ of standard solutions were derivatized at room temperature by adding $12 \mu \mathrm{l}$ of a mixture $(1: 2: 1 \mathrm{v} / \mathrm{v} / \mathrm{v})$ containing the internal standard $\left(10^{-4} \mathrm{~mol} / \mathrm{L}\right.$ cysteic acid in $0.117 \mathrm{~mol} / \mathrm{L}$ perchloric acid), a borate/ $\mathrm{NaCN}$ solution [mixed solution (100:20, v/v) of $500 \mathrm{~mm}$ borate buffer, $\mathrm{pH} 8.7$, and $87 \mathrm{~mm} \mathrm{NaCN}$ in water] and the fluorogen naphthalene-2,3-dicarboxaldehyde solution (2.925 mM in acetonitrile/water; 50:50, v/v). The electrophoretic system and optimized separation procedure were as described by Sauvinet et al. (2003). Separations were performed with a $50 \mu \mathrm{m}$ inner diameter $\times 63 \mathrm{~cm}$ fusedsilica capillary (effective length, $52 \mathrm{~cm}$ ) using $75 \mathrm{~mm}$ sodium borate containing $10 \mathrm{~mm}$ HP- $\beta$-CD and $70 \mathrm{~mm}$ SDS, $\mathrm{pH} 9.2$, as the running buffer, an applied voltage of $25 \mathrm{kV}$ and hydrodynamic injections of samples. Excitation wavelength was set at $442 \mathrm{~nm}$. Electropherograms were acquired at $15 \mathrm{~Hz}$ with P/ACE MDQ software.

Evoked EPSC recording in cortical neurons. Cortical neurons were plated on coverslips and recorded at 12-17 DIV (BX-61WI; Olympus Optical, Tokyo, Japan). The chamber was continuously perfused by the same extracellular solution as for spontaneous PSC recording $(1 \mathrm{ml} /$ $\mathrm{min})$, saturated by carbogen $\left(95 \% \mathrm{O}_{2}: 5 \% \mathrm{CO}_{2}\right)$. For internal solution, $\mathrm{Cs}^{+}$-based solution (solution 1) was used for better space clamp, and 5 mu lidocaine $N$-ethylbromide (QX314) was added to block the firing of recorded neurons. Whole-cell voltage-clamp recordings were performed

\begin{tabular}{|c|cc|rrr|}
\hline A. Genotype & \multicolumn{1}{|l|}{ Adult } & (n) & Pup & \% wt pup & $(\mathrm{n})$ \\
\hline wt & $100 \pm 11$ & $(10)$ & $21,7 \pm 0,6$ & 100 & $(11)$ \\
$+/-$ & $54 \pm 8,1$ & $(8)$ & $12,9 \pm 1,9$ & 59 & $(14)$ \\
$-/-$ & & & $5,6 \pm 1,0$ & 26 & $(11)$ \\
\hline
\end{tabular}
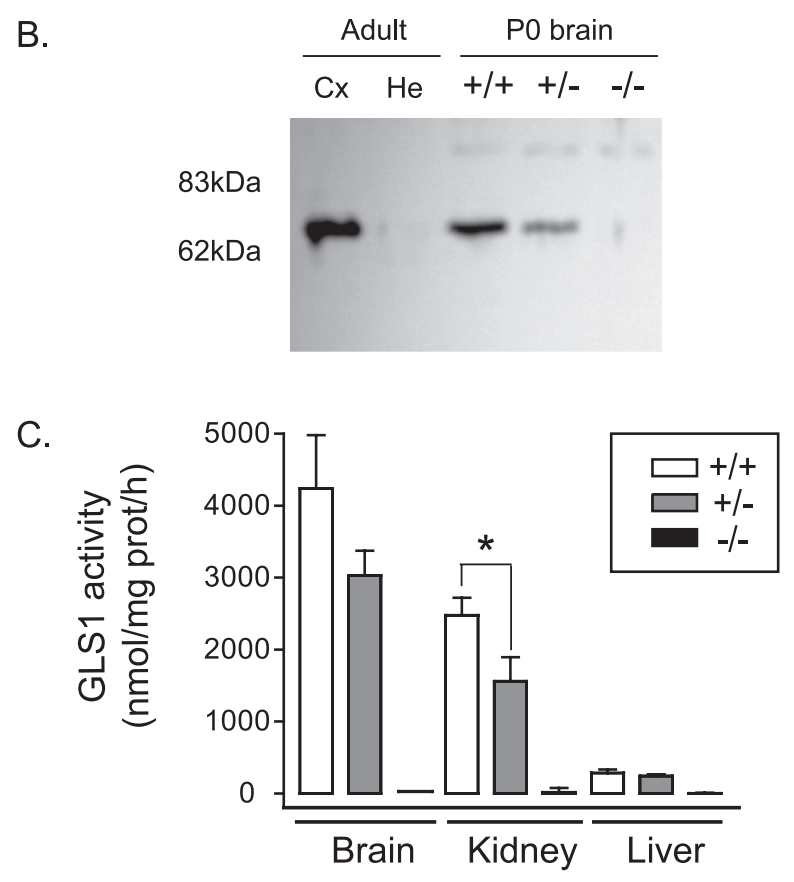

Figure 2. GLS1 expression and activity in neonates. $A$, Quantitative RT-PCR determination of GLS1 expression. mRNA expression level was normalized to the mean of adult wild-type brain $(141.8 \pm 16$, with respect to actin, which was set to 1000$)$ and expressed as a percentage (mean \pm SEM). Samples were taken from young adult (1- to 2-month-old) and late-gestation fetuses and reported for WT, heterozygous mutants $(+/-)$, and nulls $(-/-)$. A further normalization to fetal brain is shown in italics. GLS1 expression was reduced in the mutants. $\boldsymbol{B}$, Western blot analysis using anti-GLS1 antibody. Adult brain tissues show a $\sim 65 \mathrm{kDa}$ specific band in $\mathrm{C} x$ and no signal in He. For newborn brain (P0), the intensity of specific signal was strongly reduced in heterozygous $(+/-)$ compared with wild-type tissues $(+/+)$, and no signal was detected in null tissues $(-/-)$, despite the fact that five times more protein was loaded on the gel. C, GLS1 activity. Enzymatic GLS1 activity assay was measured in newborn tissues (brain, kidney, and liver). In GLS1 heterozygous pups, reduced activity was observed in the kidney ( ${ }^{*} p<0.005$ ), but activity in the brain and liver was not significantly different from WT pups. No GLS1 activity was detectable in GLS1 null tissues. Error bars represent SEM.

from large pyramidal shaped neurons with an Axopatch 200B amplifier (Molecular Devices). Series resistance (16-40 M 2 ) was compensated on-line $(60-70 \%)$. Holding potential was $-75 \mathrm{mV}$. Liquid junction potential $(\sim 12 \mathrm{mV})$ was corrected off-line. Field stimulation was delivered using a bipolar tungsten electrode (TM33CCINS; World Precision Instruments, Sarasota, FL) with pulses of $100 \mu$ s, 50-200 $\mu$ A. SR95531 and D-AP-5 were included in the extracellular solution to block $\mathrm{GABA}_{\mathrm{A}}$ and NMDA receptor responses, respectively. Data were filtered at $5 \mathrm{kHz}$ with a four-pole Bessel filter. Sampling frequency was $10 \mathrm{kHz}$. Acquisition was done with Pulse Control 4.7 (Richard J. Bookman, University of Miami, Miami, FL; http://chroma.med.miami.edu/cap/; InstruTech, Port Washington, NY) running under Igor Pro 4 (WaveMetrics, Lake Oswego, OR) on an Apple PowerMac G3 (Apple Computers, Cupertino, CA). Recorded data were analyzed off-line in Igor Pro and Axograph 4.6 (Molecular Devices).

Brainstem slice preparation and recordings. Coronal brainstem slices were obtained from newborn mice. Animals were decapitated at the C3-C4 level, and the brainstems were isolated in ice-cold artificial CSF (a-CSF) bubbled with carbogen. a-CSF composition was as follows (in $\mathrm{mm}): 128 \mathrm{NaCl}, 8 \mathrm{KCl}, 1.5 \mathrm{CaCl}_{2}, 1 \mathrm{MgSO}_{4}, 24 \mathrm{NaHCO}_{3}, 0.5 \mathrm{Na}_{2} \mathrm{HPO}_{4}$, 
30 glucose, $\mathrm{pH}$ 7.4. Using a vibratome, slices were made in the transverse plane from rostral to caudal until the axial level of the respiratory neural network was reached (Smith et al., 1991; Ramirez et al., 1996). A 500- $\mu$ m-thick slice was then removed, placed with its rostral surface up in the recording chamber maintained at $30^{\circ} \mathrm{C}$, and continuously perfused with oxygenated a-CSF. The non-NMDA glutamate receptor antagonist CNQX $(20 \mu \mathrm{M})$ was applied by perfusion. Extracellular population activity recordings were obtained using a suction electrode positioned on the surface of the slice over the pre-Bötzinger complex (PBC). The signal was amplified using a high-gain AC amplifier (Grass 7P511; Grass Instruments, Quincy, MA), bandpass filtered ( $3 \mathrm{~Hz}$ to $3 \mathrm{kHz}$ ), integrated (Neurolog System; time constant, 100 $\mathrm{ms}$ ), digitized (DigiData 1322A; Molecular Devices) to disk, and analyzed with pClamp 9 (Molecular Devices).

Statistical analysis. All results are expressed as mean \pm SEM, except for the data on mEPSCs, which are given as mean \pm SD. Overall genotype effects were documented by ANOVA, and then specific comparisons were made by $t$ tests, with Bonferroni-Dunn post hoc correction for multiple comparisons. For MDOB experiments, statistical analysis was done in SPSS (SPSS, Chicago, IL) and Systat (Systat Software, Point Richmond, CA). Repeated measures ANOVAs with test condition as the within subjects variable were used to test whether MDOBs differed by genotype (which served as the between subjects or grouping variable). To account for the often considerable litter-to-litter variation common in behavioral studies, even in inbred strains, litter number was used as a second grouping variable.

\section{Results}

\section{Generation of GLS1 mutant mice}

Via homologous recombination, we generated a mutation in the gene encoding GLS1 by inserting a floxed Neo-STOP cassette in exon $1,5^{\prime}$ of the ATG initiating codon (Fig. $1 A$ ). Southern blot analysis using a 336 bp genomic probe $5^{\prime}$ of the targeting construct (probe A) was used to identify successful recombination events in a HindIII restriction digest of ES cell DNA (Fig. 1B). Consistent with homologous recombination, probe A identified a band of genomic DNA of $5.6 \mathrm{~kb}$ in a mutant, instead of a $7 \mathrm{~kb}$ band in wild-type ES cells. This was confirmed with a specific neo probe (probe B) (Fig. $1 \mathrm{~B}$, first column) that identified the same $5.6 \mathrm{~kb}$ DNA band, and no other bands, indicating that there had been just a single recombination event. Using a PCR assay to genotype embryos and neonates (Fig. 1C) revealed a Mendelian distribution of wild-type $(+/+)$, heterozygous $(+/-)$, and null $(-/-)$ mutant genotypes (Fig. $1 D$ ), demonstrating that fetal survival was not affected by the GLS1 deletion. However, no null adult mutant could be observed.

\section{GLS1 expression and activity}

Reverse transcription (RT)-PCR analysis with primers directed to the 3' end of the GLS1 mRNA showed that transcription of the mutant gene was blocked (Fig. 2 A). In adult brain, GLS1 message was decreased to approximately one-half in GLS1 heterozygous mice; in late gestation fetuses, GLS1 expression was similarly decreased in GLS1 heterozygous fetuses and strongly reduced in bars represent SEM.

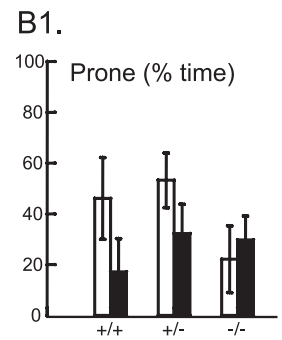

B2. B3.
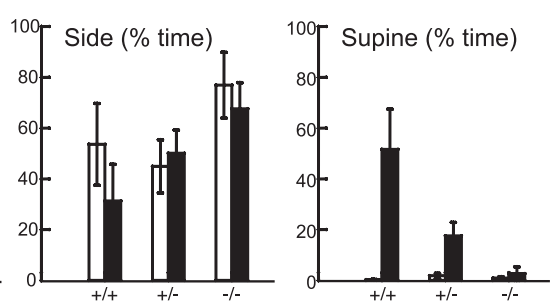

D1.

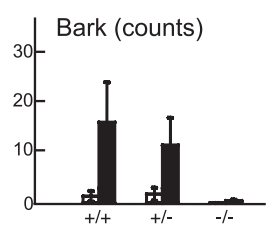

D2.

D3.

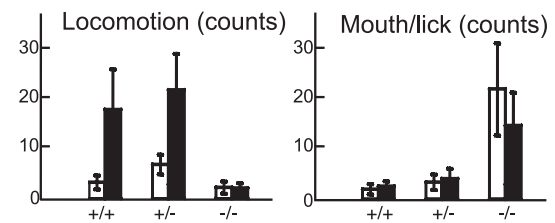

Figure 3. Maternally directed orienting behaviors in $\mathrm{P} 0$ pups. $A$, For video monitoring, pups were placed in a Plexiglas corral in ttom tier at a $45^{\circ}$ angle to provide a simultaneous ventrum-angle view of the pup; a microphone was mounted on the right side

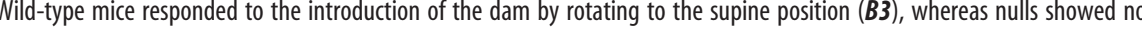
or

GLS1 null fetuses. The measured very low level of GLS1 transcription in GLS1 null pups most likely reflects background in the assay, because the combination of "false" initiating and terminating codons and splice sites in the STOP cassette completely blocks transcription (Lakso et al., 1992; Srinivas et al., 2001).

This was confirmed in Western blot analysis using a GLS1 antiserum raised against the $\mathrm{C}$ terminus of the rat enzyme (Laake et al., 1999), which is encoded by a sequence not modified in the targeted mutation. We tested the specificity of this antibody in adult brain tissues in mice; this revealed a $\sim 65 \mathrm{kDa}$ band in $\mathrm{Cx}$ but no signal in He. In wild-type pup brain, GLS1 expression was low, and we had to load five times more protein onto the gel for comparison. The intensity of the $\sim 65 \mathrm{kDa}$ GLS1 band was reduced in GLS1+/- tissue, and no signal was detected in GLS1-/- tissue (Fig. 2B).

Enzymatic assay of GLS1 in neonatal tissues (brain, kidney, and liver) revealed no activity in any tissues from GLS1 null pups; reduced activity was observed in GLS1 heterozygous pups (Fig. $2 C$ ). In neonatal liver, all GLS1 activity was caused by GLS1, which is downregulated during development, and supplanted by the liverspecific glutaminase type 2 (GLS2) in adulthood. Liver-type glutaminase (GLS2) activity is also present in neonates, but it differs in $\mathrm{pH}$ optima and sensitivity to end product inhibition (Curthoys and Watford, 1995) and thus can be assayed separately.

\section{Phenotypic characterization of GLS1 neonate mutant mice Neonatal demise}

GLS1 heterozygous mice were indistinguishable from their wildtype littermates; they developed normally and had normal reproductive functions and maternal behavior (gestation duration, litter size, and maternal feeding). In contrast, although GLS1 null neonates obtained from breeding GLS1 heterozygotes showed no 

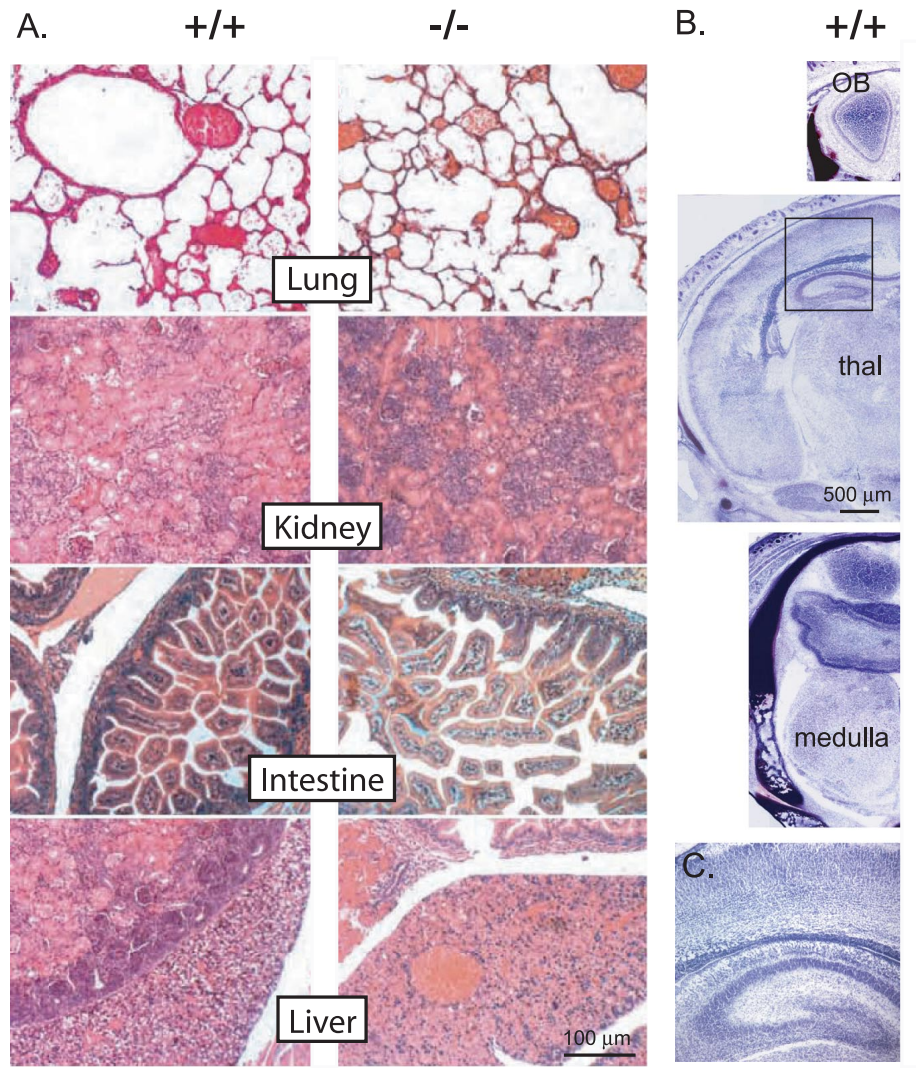

Figure 4. Normal GLS1 null mutant brain and peripheral development. $\boldsymbol{A}$, Histopathological analysis (hematoxylin, eosin, safran staining) of newborn wild-type $(+/+)$ and GLS1 null mutant $(-/-)$ periphery showed no microanatomical differences. $\boldsymbol{B}$, Photomicrograph showing Nissl-stained sections of forebrain, midbrain, and brainstem. No differences were observed in the brain size, gross structural organization, or ventricular size in GLS1 null $(-/-)$ compared with wild-type mice $(+/+)$. OB, Olfactory bulb; hip, hippocampus; cx, cerebral cortex; thal, thalamus; IC, inferior colliculi; cb, cerebellum. $C$, Enlargement of the rectangle in the second picture in $\boldsymbol{C}$ showing the normal cellular organization of hippocampal and cortical layers (cortical plate, layers $\mathrm{V}$ and $\mathrm{VI}$ ) in GLS1 null mice.

gross physical or behavioral abnormalities, none survived for longer than $36 \mathrm{~h}$ after birth. GLS1 null pups showed no abnormal distinguishing behaviors. They were able to right themselves properly after being placed on their back. However, a few hours after birth, when wild-type and heterozygous littermates had nursed, null mutants were easily distinguished by the lack of a milk band. In fact, they rarely had milk in their stomach (and, if any, in small amount) and became dehydrated. However, death did not result from starvation, because unfed wild-type and heterozygous littermates all survived beyond $36 \mathrm{~h}$, the maximal life span of the GLS1 null pups. GLS1 null pups were not abandoned, because they were found intermixed with other littermates under the dam. Manual stimulation of the mouth region or dropper feeding evoked rhythmic jaw movements in the null pups, and they would suckle a feeding tube. Decreased feeding was therefore not a result of gross defects in facial structure or the ability to suckle. Once pups had begun nursing, litters were culled to include pups without milk bands (presumptive null mutants) and two pups with milk bands (presumptive heterozygotes or WT littermates) to maintain lactation in the dam. Even with favored access to the dam, GLS1 null pups did not survive any longer, indicating that the demise of GLS1 null pups was not a result of the inability of these mutants to compete with more fit littermates.

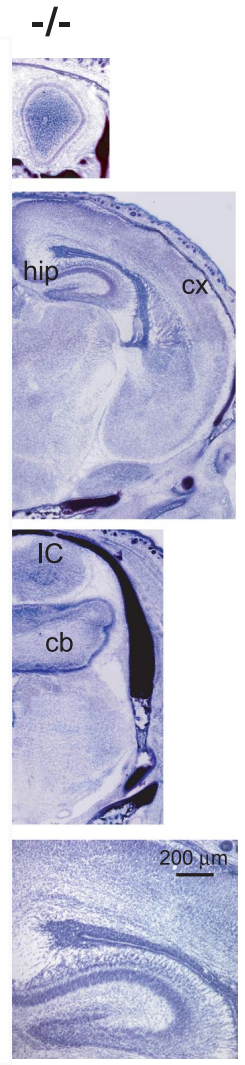

Maternally directed oriented behavior

The goal-directed behavior of the GLS1 null pups was not normal. Normally, neonates must seek and maintain close contact with their mother to maintain body temperature and gain nourishment. Recent work has identified a suite of MDOBs by which neonatal rodents seek, achieve, and maintain close contact with their dam's ventrum and position themselves to grasp a nipple (Stern, 1997; Polan et al., 2002). We observed 29 P0 pups from six litters. Pups had the chance to nurse at least once, as evidenced by the presence of milk bands. Pups were placed individually in a nest-temperature plastic corral (Fig. $3 A$ ) and videotaped for $4 \mathrm{~min}$, first alone (control condition) and then for an another $4 \mathrm{~min}$ with the anesthetized dam (test condition). In the control condition, the pup was placed prone in the corral and could move about freely; when tested with the dam, the pup was again placed prone inside the corral and the anesthetized dam, facing ventrum-downward, was lowered gently onto a supporting scaffold above the corral so that her ventral surface came into light contact with the dorsal surface of the pup, initiating the 4 min test. This arrangement creates a naturalistic encounter between the pup and the dam, which mimics the hovering position over the litter pile assumed by lactating dams. Pups were scored blinded to genotype for wakeful behaviors, including MDOB and nipple grasp using the scoring method of Polan et al. (2002). Two wild-type pups and one heterozygote pup (but no null pups) grasped a nipple during the $4 \mathrm{~min}$ test; the mean latency to grasp was $12 \mathrm{~s}$. Because nipple grasp affects the subsequent behaviors of pups, these three pups were not included in the subsequent analysis.

Of the 26 pups scored, the most striking difference was a massive reduction compared with wild type in the proportion of time that GLS1 null pups spent supine, specifically under the dam's ventrum (Fig. $3 B$ ). In particular, the null pups spent only $2.6 \pm$ $2.7 \%$ of their 4 min encounter with the dam in the supine orientation; the heterozygotes spent $17.6 \pm 5.3 \%$ of their test time supine, whereas the wild-type pups spent fully $52.0 \pm 15.8 \%$ of their time supine (main effects of genotype and test condition, $F=7.49$ and $20.30 ; d f=2$ and $1, p<0.0005$, respectively; interaction of genotype and test condition, $F=8.36, d f=2, p<$ $0.005)$. The reduction in the amount of time spent supine by nulls was mostly compensated for by an excess in the amount of time the nulls spent in the on-side orientation (main effect of genotype, $F=3.88 ; d f=2 ; p<0.05)$. Without supination, pups cannot achieve ventrum-to-ventrum contact with the dam and are therefore not positioned to efficiently gain warmth from the dam or locate and grasp a nipple to initiate suckling. Thus, the deficits in supination seen in the heterozygotes and the nulls suggest an impairment in their ability to orient to their mothers, the severity of which is GLS1-genotype dependent.

Barking and locomotion under the dam's ventrum were also 
reduced in the nulls (Fig. 3D1,D2). The nulls locomoted a mean of just $2.2 \pm 0.1$ times and barked a mean of only $0.44 \pm 0.26$ times, compared with $22.1 \pm 7.0$ and $18.1 \pm 8.1$ locomotions for heterozygotes and wild types, respectively, and $11.6 \pm 5.5$ and $16.1 \pm 8.0$ barks for heterozygotes and wild types, respectively (for locomotion main effects of genotype and test condition, $F=$ 4.65 and 9.54, $d f=2$ and 1 , respectively, both $p<0.05$; for barking main effects of genotype and test condition, $F=3.48$ and $8.46, d f=2$ and $1, p=0.053$ and $<0.05$, respectively; interaction of genotype and test condition for locomotion, $F=2.61, d f=2$, $p=0.10$, and for barking, $F=3.42, d f=2, p=0.055)$.

Evidently, the reductions by the nulls in time spent supine, locomotion, and barking under the dam's ventrum were not simply a result of a generalized reduction in arousal or activation. There were no significant differences across the genotypes in the frequencies of head, limb, or torso movements nor the frequency of change of dorsoventral orientation. However, mouthing/licking, possibly a neonatal correlate of vacuous chewing, was markedly increased (Fig. 3D3). The nulls mouthed/licked $14.9 \pm 6.6$ times while under the dam, compared with $4.1 \pm 2.1$ times by the heterozygotes, and $2.6 \pm 1.0$ times by the wild types (main effect of genotype, $F=7.18, d f=2, p<0.05$ ). Mouthing/licking was not affected by test condition or the interaction of genotype and test condition. A quantitative index of general arousal or activation was computed for each pup in each test as the sum of the frequencies of all awake behaviors (Fig. 3C), which showed no differences among the genotypes or between the test conditions, nor an interaction of genotype with test condition. Thus, the nulls are capable of movement but are deficient in normal, neonatal, goal-directed behavior.

\section{Anatomy}

Comparison of the body weight of wild type (1.42 $\pm 0.02 \mathrm{~g} ; n=$ $35)$, heterozygous ( $1.42 \pm 0.02 \mathrm{~g} ; n=54)$, and null $(1.30 \pm 0.03 \mathrm{~g}$; $n=37$ ) newborns revealed null mutant weighed $8.5 \%$ less than controls $(+/+$ and $+/-$ combined; $p<0.05)$. To determine whether GLS1 null pups were just smaller or had more serious defects, we analyzed whole-body coronal sections stained with hematoxylin, eosin, and safran. Qualitative assessment performed on thoracic and abdominal sections of E12-E18 embryos and newborn mice (P0) revealed no differences between genotypes, in gross anatomy or histology of the brain, spinal cord, lung, heart, esophagus, trachea, stomach, pancreas, liver, intestine, kidney, spleen, bone, or fat tissues (P0) (Fig. 4A). The brain at birth showed no abnormalities in size, structural and cellular organization, or in ventricular size (Fig. $4 B, C$ ).

\section{Respiratory function}

During baseline breathing in room air, minute-ventilation $\left(V_{\mathrm{E}}\right)$, breathing frequency $\left(f_{\mathrm{R}}\right)$, and tidal volume $\left(V_{\mathrm{T}}\right)$ were not significantly different between wild-type and heterozygous animals, but null mice hypoventilated (Fig. $5 A c, B$, left panel). $V_{\mathrm{E}}$ was $40 \%$ lower than in wild-type mice $(p<0.05)$; this lower ventilation essentially resulted from a lower $V_{\mathrm{T}}(-29 \% ; p<0.05)$. On the first day of life, neonates normally exhibit apneic periods (Jacquin et al., 1996), with large variations between animals and in the same animal over successive records. Apneic respiration, expressed as average percentage of recording time, was not significantly different in wild-type $(7.2 \pm 2.1 \%)$ and heterozygous $(3.5 \pm 2.0 \%)$ mice but was significantly higher in null mutants (13.8 $\pm 2.3 \% ; p<0.05$ vs the other two genotypes). Hypercapneic challenge equally increased $V_{\mathrm{E}}$ in both wild-type and heterozygous animals (for both, $p<0.01$ ) (Fig. $5 A b$ ) through in-
A.

AIR

$6 \% \mathrm{CO}_{2}$

a

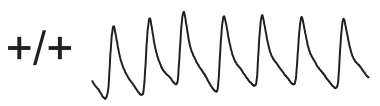

b

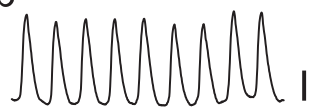

- ${ }^{c}$ Navan

d

MMMMI

$1 \mathrm{sec}$

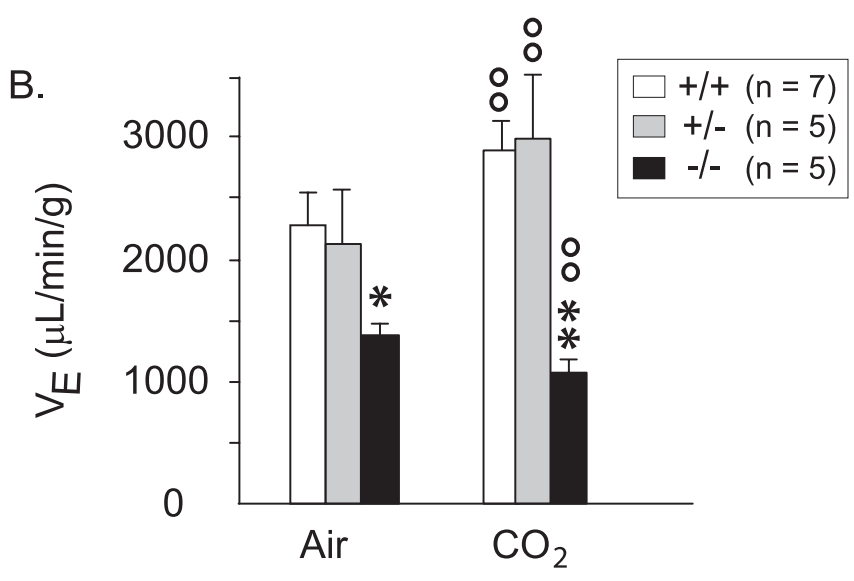

Figure 5. Respiratory function of newborn GLS1 mutants. $A$, Plethysmographic recordings showing baseline respiration in a wild-type $(+/+)$ and a null mutant $(-/-)$ mouse breathing room air $(\boldsymbol{a}, \boldsymbol{c})$ and ventilatory responses $(\boldsymbol{b}, \boldsymbol{d})$ to a hypercapneic challenge $\left(6 \% \mathrm{CO}_{2}\right)$. Vertical calibrations: $10 \mu$, inspiration up. $\boldsymbol{B}$, Minute ventilation $\left(V_{\mathrm{E}}\right)$ in wild-type, heterozygous, and null mutant mice inhaling room air or $6 \% \mathrm{CO}_{2} .{ }^{*} p<0.05$; ${ }^{* *} p<0.01$ versus wild-type mice; ${ }^{\circ} p<0.01$ versus baseline air breathing. GLS1 $-/-$ pups hypoventilated and showed a paradoxical hypercapneic response. Error bars represent SEM.

creases in both $V_{\mathrm{T}}$ and $f_{\mathrm{R}}$. However, in null mutants, $V_{\mathrm{E}}$ decreased by $22 \%$ during $\mathrm{CO}_{2}$ breathing compared with air breathing (Fig. $5 B)$. This was attributable to a profound decrease in $V_{\mathrm{T}}(-30 \%$; $p<0.02$ ), whereas $f_{\mathrm{R}}$ was slightly but significantly increased $(+9 \% ; p<0.05)($ Fig. 5Ad,B). As a result, with hypercapnia, both $V_{\mathrm{E}}$ and $V_{\mathrm{T}}$ were paradoxically much lower in GLS1 null mice $(p<0.01-0.001)$ (Fig. 5Ad,B, right panel). Apneic respiration decreased in all genotypes with hypercapnia but remained significantly higher in null mice ( $9 \pm 2 \%$ of recording time) compared with the other genotypes ( $p<0.01$ for both genotypes), in which periods of apnea nearly disappeared $(<2.5 \%$ of recording time). After 2 min of hypercapnia, three of the five GLS1 null mice showed short periods of "gasping" inspiration, sometimes intermingled with eupneic breaths, a pattern that was never seen in the other genotypes.

\section{Glutamatergic synaptic transmission}

Because of the neonatal demise, we used primary cultures of cortical neurons isolated from E18 brain embryos to examine excitatory neurotransmission. Cultures comprised $\sim 80 \%$ neurons. Glial fibrillary acidic protein (specific marker of glial cells) labeling showed each well also contained astrocytes (data not shown) that presumably supported neuronal survival and would contribute to maturation of synapses. At 6-21 DIV, neurons from wildtype embryos expressed GLS1 (Fig. 6). GLS1 labeling superimposed on mitochondrial labeling (Fig. 6C), showing that GLS1, which is a mitochondrial enzyme (Laake et al., 1999; Kvamme et 


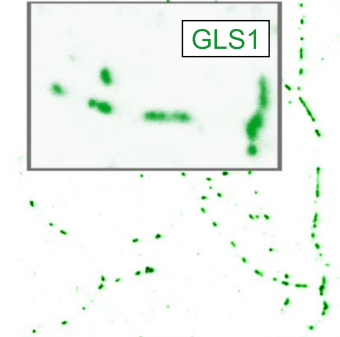

A
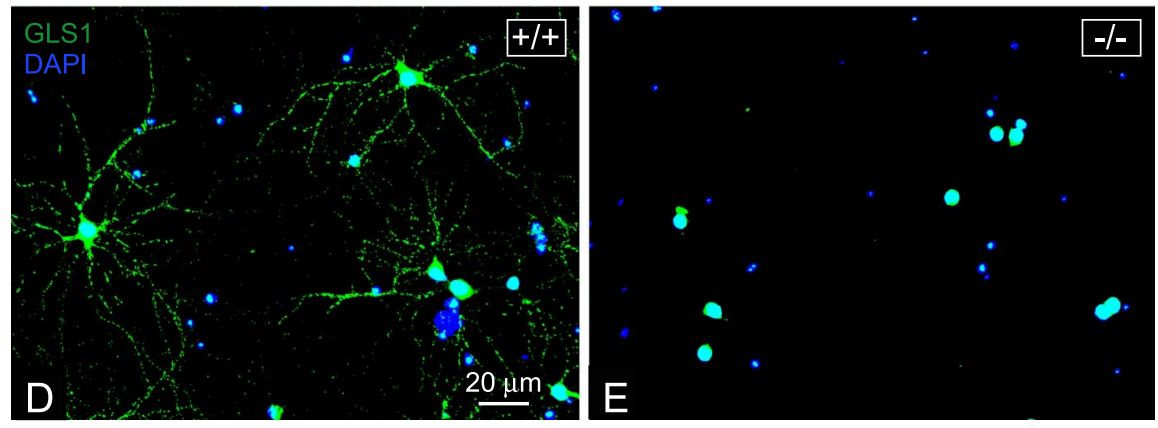

Figure 6. GLS1 expression in cortical neuron primary culture. $\boldsymbol{A}-\boldsymbol{C}$, Mitochondrial GLS1 labeling. Confocal immunofluorescence detection of labeling performed with anti-GLS1 $(\boldsymbol{A})$ and anti-mitochondria $(\boldsymbol{B})$ antibodies. Pictures were inverted and colors were indexed to show GLS1 in green and mitochondria in red. Colocalization of GLS1 and mitochondria labeling is shown in $\boldsymbol{C}$. In each panel, an enlargement $(5 \times)$ of the staining is shown in the top left, corresponding to a portion of the picture represented by the gray rectangle. GLS1 and mitochondria pictures were slightly shifted to show clearly the coincidence of staining patterns. $\boldsymbol{D}, \boldsymbol{E}$ Fluorescence detection of GLS1 expression with Alexa 488-conjugated anti-rabbit antibodies (green) and nuclear cell localization detected with DAPI (blue). The mitochondrial GLS1 immunofluorescence observed in wild-type culture (D) was absent in GLS1-/- neurons $(\boldsymbol{E})$. Residual GLS1 nuclear labeling was evident, but this has been shown previously to be nonspecific (Laake et al., 1999).

al., 2000), exhibited the expected subcellular distribution. Neurons from null embryos $(-/-)$ displayed no GLS1 labeling (Fig. $6 E$ ), whereas neurons from heterozygous embryos showed an intermediate phenotype with a reduction in GLS1 labeling (data not shown).

In cultures of GLS1+/+ and GLS1-/- neurons, spontaneous synaptic currents were recorded in whole-cell configuration. At a holding potential near resting level $(-70 \mathrm{mV})$, spontaneous inward currents with fast or slow kinetics were recorded (Fig. 7A, left bottom panel). The fast synaptic currents were identified as EPSCs, because they reversed near $0 \mathrm{mV}$ and were blocked by the AMPA/kainate receptor antagonist CNQX $(10 \mu \mathrm{M})$. The slow synaptic currents were identified as $\mathrm{GABA}_{\mathrm{A}}$-mediated IPSCs with a reversal near $-30 \mathrm{mV}$ (the chloride equilibrium under our recording conditions) and were blocked by the $\mathrm{GABA}_{\mathrm{A}}$ receptor antagonists bicuculline $(10 \mu \mathrm{M})$ (Fig. $7 A$, right panels) or gabazine (SR95531; $10 \mu \mathrm{M})$.

Recent evidence indicates that the postsynaptic AMPA receptors are not saturated following the single-site release of glutamate (Auger and Marty, 2000). Therefore, efficacy of glutamatergic transmission depends on the amount of glutamate released. In fact, it has been shown that the amplitude of the TTX-resistant mEPSCs depends on the glutamate content of the presynaptic terminals (Ishikawa et al., 2002). To test the possibility that the synaptic terminals of GLS1-/- neurons contain less glutamate than the GLS1+/+ terminals, we compared the amplitude and the kinetics of the mEPSCs (Fig. $7 B, C$ ). We found no difference in the two parameters $(27.89 \pm 6.84 \mathrm{pA}, n=7$ and $26.87 \pm 4.49$ $\mathrm{pA}, n=8$; and $2.45 \pm 0.36 \mathrm{~ms}, n=7$ and $2.79 \pm 0.61 \mathrm{~ms}, n=8$ in the GLS1+/+ and the GLS1-/- neurons, respectively). These results suggest that the lack of GLS1 does not impact single-release events.

At 7 DIV and onward, cortical neurons in culture displayed spontaneous spike discharge and synaptic currents. Thus, the release of glutamate and GABA was examined at 14-15 DIV. Basal GABA and glutamate release was first determined during $15 \mathrm{~min}$ to access their ability to release during this timeframe. Both cultures prepared from wild-type or GLS1 null embryos released GABA (Fig. 8A1) and glutamate (Fig. 8A2). The effect of depolarization by $56 \mathrm{~mm} \mathrm{~K}^{+}$(high $\mathrm{K}^{+}$) on GABA and glutamate release was examined during two 3 min epochs followed by a return to low $\mathrm{K}^{+}(5 \mathrm{mM})$. Depolarization resulted in a large increase in GABA and glutamate release (twofold to fourfold) in GLS1+/+ and GLS1-/- cultures. Evoked glutamate release was significantly lower in GLS1 - / - cultures with a deficit of $28.5 \%$ during the first stimulation (Fig. 8 A2, column 2) and $42.3 \%$ during the second (Fig. 8A2, column 4), whereas GABA release was unchanged (Fig. 8A1). Evoked release was $\mathrm{Ca}^{2+}$ dependent, whereas basal release $\left(5 \mathrm{~mm} \mathrm{~K}^{+}\right)$ was $\mathrm{Ca}^{2+}$ independent (data not shown), suggesting that basal release was caused by nonsynaptic mechanisms, possibly reverse transport (Danbolt, 2001), and thus would reflect diminished cytoplasmic glutamate levels. Consistent with this idea, we observed that basal $\mathrm{Ca}^{2+}$-independent glutamate release, but not GABA release, was diminished in GLS1-/- compared with GLS1+/+ cultures (Fig. 8A2, columns $1,3,5)$.

To examine the effect of the GLS1 knock-out on glutamatergic synaptic transmission more directly, we recorded from single neurons in whole-cell patch configuration and stimulated neighboring neurons in the presence of gabazine $(10 \mu \mathrm{M})$ and the NMDA blocker D-AP-5 $(50 \mu \mathrm{M})$ to isolate the AMPA/kainate receptor-mediated EPSC. The amplitude of the evoked EPSC was not different in GLS1 - / - cultures. However, with repeated field stimulation at $10 \mathrm{~Hz}$ for $20 \mathrm{~s}$, the EPSC decremented exponentially. The decrement was several-fold faster in GLS1-/- cells (Fig. $8 \mathrm{~B}$ ). In contrast, there was no genotypic difference in the evoked GABAergic field IPSC with $10 \mathrm{~Hz}$ stimulation. Nor did we find any difference between GLS1-/- and WT cultures in the average amplitude of the first three IPSCs (GLS1-/-, $156.2 \pm$ $25.1 \mathrm{pA}, n=13$ cells; WT, $206.8 \pm 42.2 \mathrm{pA}, n=13$ cells; $p=0.30$, unpaired $t$ test), the average amplitude of the last 30 IPSCs (GLS1-/ $-, 16.0 \pm 2.2 \mathrm{pA}, n=13$ cells; WT, $21.4 \pm 6.3 \mathrm{pA}, n=$ 13 cells; $p=0.43$, unpaired $t$ test), or the decay time constant (GLS1-/-, $0.70 \pm 0.12 \mathrm{~s}, n=13$ cells; WT, $0.85 \pm 0.37 \mathrm{~s}, n=13$ cells; $p=0.69$, unpaired $t$ test). Thus, neurons lacking GLS1 show apparently normal, unaltered baseline glutamatergic synaptic activity but are less resilient when activated at physiological frequencies. Therefore, the GLS1 deficit should principally impact more active neural circuits. 
Effect of GLS1 mutation on the glutamate-dependent rhythmic activity generated by an active neural network in vitro

In a final set of experiments, we examined the presumed impact of the GLS1 deletion on rhythmic activity in the $\mathrm{PBC}$ in brain slices made from the ventrolateral medulla. The PBC is known to contain the brainstem respiratory generator and relies mainly on excitatory glutamatergic connections (Funk et al., 1993), without significant contribution of chloridemediated inhibitory connections in newborn mice (Feldman and Smith, 1989; Ritter and Zhang, 2000). The PBC can be isolated in vitro in a transverse slice and continues to generate the spontaneous rhythmic activity that drives respiratory motor output, although at a lower frequency than in vivo (Smith et al., 1991). Extracellular field recordings showed that the PBC network was functional in GLS1 mutants (Fig. 9A, left). However, the frequency of respiration-associated bursts was significantly higher in null mutants (Fig. 9A, left panel). Blockade with CNQX $(20 \mu \mathrm{M})$ suppressed rhythmic activity in wild-type controls as well as in null mutants (Fig. 9A, compare control and CNQX), and this effect was reversible (Fig. $9 A$, wash out), indicating that in GLS1 null mutants, the respiratory rhythm is present and still depends entirely on glutamatergic transmission, although its frequency is altered. These results confirm that the control of respiratory frequency depends on the integrity of glutamatergic transmission in the respiratory network. Apparently, even a modest alteration in glutamatergic transmission can change neuronal rhythms significantly with profound consequences.

\section{Discussion}

We addressed the importance of GLS1 in the enzymatic provision of neurotransmitter glutamate by knocking out the gene in mice. GLS1 null mutants failed to feed because of deficits in goal-directed behavior and died within the first postnatal day with a phenotype of altered respiration. In culture, cortical neurons from GLS1 null mutants showed normal mEPSCs; however, evoked glutamate release was diminished, and when synapses were driven at physiological frequencies, they were more quickly exhausted. Despite the conclusions of many previous studies that GLS1 is the principal source of neurotransmitter glutamate, knocking out the enzyme had a surprisingly subtle effect on glutamatergic synaptic transmission. However, it per-

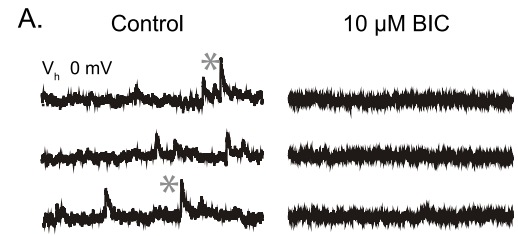

B.

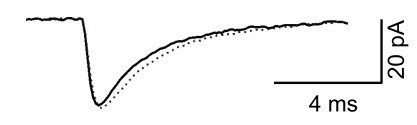

C1.

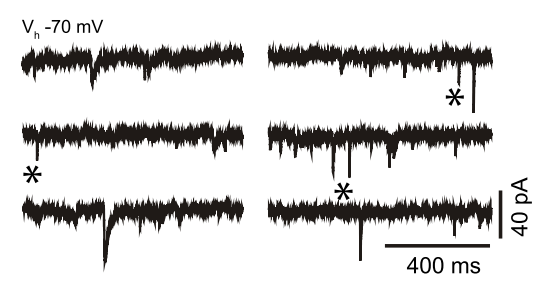

C2.

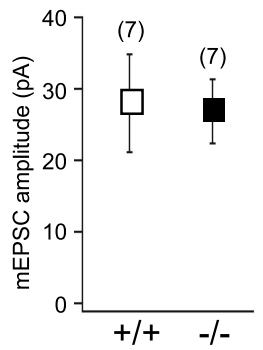

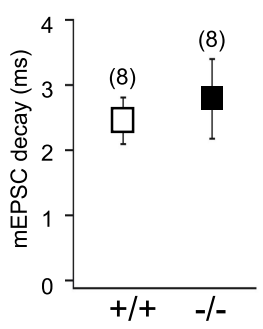

Figure 7. Spontaneous EPSCs and IPSCs in GLS1+/+ and GLS1 - / - cortical neurons in culture. A, In a GLS1 - / - neuron (13 DIV), spontaneous synaptic currents were recorded under control conditions and after perfusion of $10 \mu \mathrm{m} \mathrm{BIC}$ at two holding potentials: at $0 \mathrm{mV}$, near the reversal potential of the EPSCS, and at $-70 \mathrm{mV}$, near the resting potential. At $0 \mathrm{mV}$, the slow outward

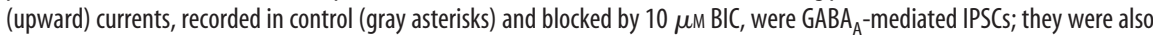
recorded at $-70 \mathrm{mV}$ as slow inward (downward) currents seen in control but not in BIC. The faster inward currents (black asterisks) that were recorded at $-70 \mathrm{mV}$ and persisted in BIC were spontaneous glutamatergic AMPA receptor-mediated EPSCs. B, Superimposed average of mEPSCS $(n=100)$, recorded in $1 \mu \mathrm{m}$ TTX and $10 \mu \mathrm{m}$ SR95531, from GLS1 + / + (black trace) and GLS1 - / - (dotted trace) neurons showed no genotypic difference. $\mathbf{C 1}, \mathbf{C}$, The amplitude (C) and the decay (C2) of the mEPSCs (measured in TTX and SR95531) showed no genotypic difference. Error bars represent SD.
A1.

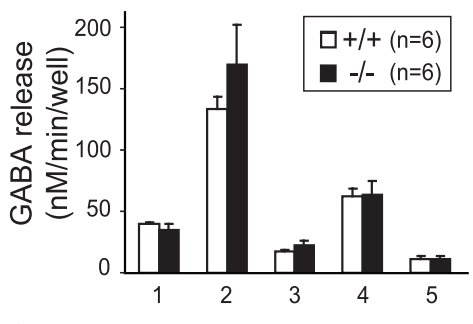

high $\mathrm{K}+-++\quad+$
A2.

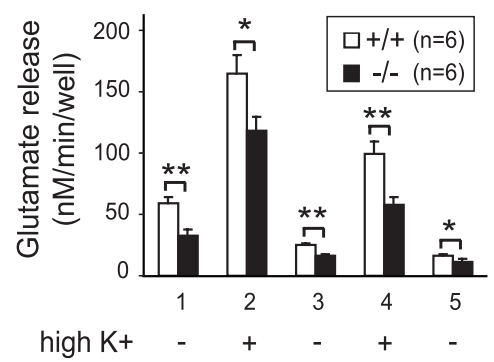

B1.
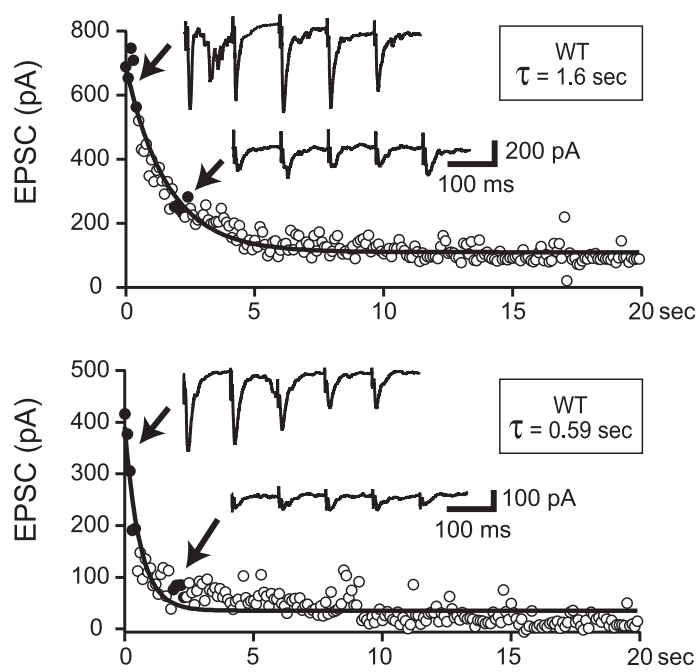

B2. Decay $\tau$ with $10 \mathrm{~Hz}$ stim

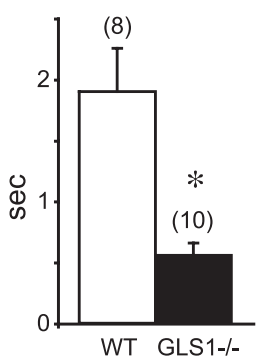

Figure 8. Neurotransmission in cultured cortical neurons. A1, $\mathbf{A 2}, \mathrm{GABA}(\boldsymbol{A 1})$ and glutamate (A2) basal and evoked release. Columns 1, 3, and 5 represent basal release $(5 \mathrm{~mm} \mathrm{KCl})$. Evoked release with $56 \mathrm{~mm} \mathrm{KCl}$ was performed during the 3 min corresponding to columns 2 and 4 . Each column represents amino acid concentration released/minute, pooled by three fractions, with triplicate determinations per animal. ${ }^{*} p<0.05 ;{ }^{* *} p<0.01$. B1, B2, Decay of EPSCs during electrical stimulation. Field stimulation was delivered at $10 \mathrm{~Hz}$ for $20 \mathrm{~s}$ (200 stimuli) and EPSCs recorded in whole-cell mode. The intracellular solution contained QX314 to prevent spiking. Graphs show the decline of EPSC amplitude during $10 \mathrm{~Hz}$ stimulation in wild-type (B1, top) and GLS1-/- (B1, bottom) cells. EPSC traces are shown for closed circles. The decay followed a single exponential ( $\tau=$ time constant). The decay time constant was significantly shorter in GLS1 - / - neurons (B2). Neither the average amplitude of the first three EPSCs nor the average amplitude of the last 30 EPSCs showed significant genotypic variation (data not shown). Numbers of recorded cells are indicated in parentheses. Data were obtained from three sets of matched cultures. ${ }^{*} p<0.01$. Error bars represent SEM. 
A.

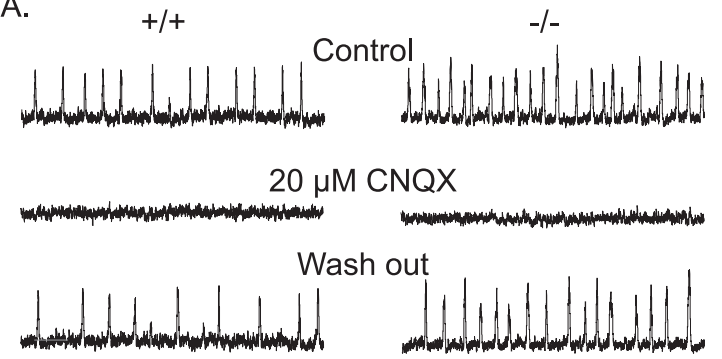

B.

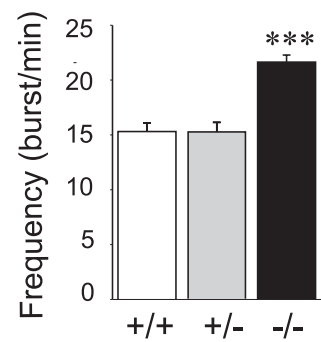

(7) (8) (7)

Figure 9. Rhythmic activity generated by the respiratory neural network in vitro in coronal brainstem slices. $A$, Integrated population activity recorded in preparations obtained from wild-type $(+/+)$ and null $(-/-)$ mutants in control conditions (top traces) after blockade of glutamatergic connections (middle traces) and after wash out (bottom traces). Bath application of $20 \mu \mathrm{M}$ CNQX abolished spontaneous rhythmic activity in both wild-type and null mutant slices. $\boldsymbol{B}$, Mean frequency of rhythmic activity. There was a significant increase in respiration-associated burst frequency in null mutant slices $(-/-)$. Numbers of animals are indicated in parentheses. ${ }^{* * *} p<0.001$ nulls versus wild type and heterozygotes. Error bars represent SEM.

turbed glutamatergic neural networks such as brainstem respiratory circuits that depend on robust summation of synaptic inputs and thus could account for the altered respiration phenotype.

\section{Persistence of glutamatergic activity in GLS1 null mutants}

mEPSC amplitude was not reduced in cultured GLS1 - / - cortical neurons, suggesting that there were adequate levels of intrasynaptic glutamate to load vesicles under conditions of basal activity. Similarly, field stimulation at low frequencies showed no impact of the GLS1 deletion on evoked EPSC amplitude. Moreover, glutamatergic neuronal networks, such as those generating the respiratory rhythm in the PBC, remained functional in GLS1 null mutants. Therefore, under basal conditions, GLS1-/- neurons showed normal glutamatergic synaptic function. However, basal glutamate release was strongly reduced in GLS1-/- culture. Because GLS1 is only expressed in neurons, presumably this reduction reflects a reduction in cytoplasmic glutamate levels, given that glutamate produced by GLS1 must traverse the cytoplasm before being taken up into vesicles. Indeed, in preliminary magnetic resonance spectroscopic determinations of glutamate levels in brains of P0 GLS1-/- pups, we found that there is an $\sim 50 \%$ reduction in glutamate levels compared with WT brains, with no reduction in GABA levels (Galloway et al., 2005). The persistence of glutamatergic synaptic transmission in GLS1 null mutants can be accounted for by other glutamate-synthetic pathways that have long been known in neurons such as transamination of $\alpha$-ketoglutarate with alanine, branched-chain amino acids, or lysine (Papes et al., 2001; Hertz, 2004; Sweatt et al., 2004; Yudkoff et al., 2005). Direct reuptake of glutamate into neurons (Chen et al., 2004; Waagepetersen et al., 2005) could also account for relatively normal glutamatergic synaptic function in the absence of GLS1.

\section{Altered functioning of glutamatergic neural networks in GLS1 null mutants}

Evoked glutamate release from GLS1-/- cortical neurons in culture was reduced, providing an estimate of the relative importance of the GLS1 pathway (28-42\%). When synapses were stimulated at a sustained rate, glutamatergic synaptic transmission was exhausted more rapidly in GLS1-/- neurons. Presumably, the readily releasable synaptic vesicle pool was smaller, or presynaptic mechanisms were altered in GLS1-/ - neurons because of the GLS1 deficit. The diminished $\mathrm{Ca}^{2+}$-independent basal glutamate release in GLS1-/- neurons, presumably because of nonsynaptic mechanisms, together with the diminished overall glumatergic synaptic transmission at $\mathrm{P} 0$, because chloridedependent inhibitions develop later (Feldman and Smith, 1989). PBC cells discharge intensely during each inspiratory phase (Thoby-Brisson and Ramirez, 2000). The increased PBC respiratory frequency in slices from null mutants probably results from the deficit in glutamatergic transmission, although an indirect effect of the mutation on the network cannot be entirely ruled out. Modeling the respiratory network reveals that excitatory synaptic transmission and respiratory frequency are inversely related (Butera et al., 1999). The hypothesis was that weaker synaptic currents induce weaker activation of conductance involved in burst generation. Then, burst termination requires a weaker current inactivation, leading to a shorter recovery from inactivation, and higher burst frequency. Predictions of this model have been tested in two recent in vitro studies using different approaches. Del Negro and colleagues (2001) demonstrated that decreasing the excitatory synaptic coupling within the network by removing inputs coming from the contralateral network resulted in an increase in respiratory frequency. Conversely, enhancement of the glutamatergic synaptic drive, as a result of exposure to brain-derived neurotrophic factor, decreased the frequency of rhythmic activity (Thoby-Brisson et al., 2003).

Tidal volume, which requires robust summation of glutamatergic synaptic inputs to generate the inspiratory ramp-like motor output, was severely affected in vivo. Altered tidal volume was the main cause of hypoventilation in vivo, and it was not compensated for by an increase in respiratory rate. Chemosensitivity to carbon dioxide was also affected. The inability of GLS1 null mutants to increase ventilation in response to a chemosensory challenge suggests that the baseline synaptic activity of respiratory neurons was already at maximum, without the normal reserve. This impaired reactivity can be related to the exhaustion of glutamatergic transmission after physiological stimulation that we observed in GLS1-/ - cortical neuron cultures. Alternatively, the response to $\mathrm{CO}_{2}$ might have been blunted by dysfunction of glutamatergic neurons in chemosensory areas near the ventral surface of the medulla (Mulkey et al., 2004; Weston et al., 2004). In either case, the hypoventilation phenotype is consistent with the glutamate release deficit demonstrated in vitro.

\section{Demise of GLS1 pups}

Newborn GLS1 null pups invariably died during the first postnatal day. Although alternative pathways might have been upregulated during gestation, the results show clearly that normal GLS1 function is essential for the proper functioning of glutamatergic 
networks of vital importance. GLS1 also plays a key role in supplying metabolic energy in the developing embryo by catabolizing amino acids for energy production in liver, kidney, and small intestine (Curthoys and Watford, 1995), so its absence could explain the smaller size of the GLS1 null mutants at birth but probably not their early demise, because there were no microscopic abnormalities in the organs of GLS1 null pups. This leaves the respiratory dysfunction as the probable cause of their early demise. Renal GLS1 expression normally increases dramatically with acidosis, driving the excretion of $\mathrm{NH}_{4}{ }^{+}$(Curthoys, 2001). Lacking this compensatory mechanism, null mutants would develop a respiratory acidosis. This would explain the increase in gasping seen in the mutant pups and argues for uncompensated respiratory acidosis as the proximate cause of their early demise.

\section{Impact of GLS1 knock-out on neonatal behavior}

Some of the behavioral abnormalities observed in the GLS1 null pups could be related to their respiratory abnormalities. For example, increased mouthing/licking might be attributable to abnormal respiration, whereas the decrease in barking might be attributable to the impact of the respiratory compromise on the ability to vocalize. However, it seems unlikely that this would explain all of the altered behavior of the nulls, such as the dramatic decrease in supination. Thus, it would appear that the altered behavior of the GLS1 null pups arose at least in part from nonrespiratory mechanisms, possibly because of deficits in the central processing of sensory input or in the organization of goaldirected behavior. The GLS1 null pups showed a normal level of behavioral activity, but it was disorganized: the pups did not orient to the dam, never succeeded in grasping a nipple, and did not reliably obtain milk. It seems plausible that the deficits in MDOBs interfere with the ability of the null pups to locate and grasp their dams' nipples. Null pups did suckle a feeding tube, indicating that they were capable of orally grasping an object. Although, starvation does not explain their demise, starvation and/or dehydration resulting from the failure to nurse could make the null pups more vulnerable to the effects of respiratory compromise. Just as the respiratory rhythm is abnormal in the GLS1-/- brainstem, one may surmise that the overall frequencies or organization of regular neuronal rhythms responsible for the integration of brain function (Engel and Singer, 2001) are also impaired, accounting for the disorganized behavior.

\section{Implications and conclusion}

The GLS1 mutant mice we have made will permit tissue-specific rescue, because the STOP cassette we inserted to block GLS1 transcription is flanked by lox $\mathrm{P}$ sites. Therefore, breeding the GLS1 mutant mice with tissue-specific cre recombinaseexpressing mice should restore GLS1 expression selectively (Ghosh and Van Duyne, 2002). Rescuing GLS1 in brainstem respiratory neurons or in kidney would provide means to prove that the early demise of the mice is a result of respiratory failure or uncompensated respiratory acidosis. At this point, we conclude that GLS1 null mutants have an activity-dependent deficit in glutamatergic synaptic transmission resulting in altered respiratory rhythms and early goal-directed behavior. Although alternative synthetic pathways allow temporary postnatal survival, the mice breathe poorly and die, indicating that GLS1 function is essential for survival.

\section{References}

Auger C, Marty A (2000) Quantal currents at single-site central synapses. J Physiol (Lond) 526:3-11.
Bai L, Xu H, Collins JF, Ghishan FK (2001) Molecular and functional analysis of a novel neuronal vesicular glutamate transporter. J Biol Chem 276:36764-36769.

Banner C, Hwang JJ, Shapiro RA, Wenthold RJ, Nakatani Y, Lampel KA, Thomas JW, Huie D, Curthoys NP (1988) Isolation of a cDNA for rat brain glutaminase. Brain Res 427:247-254.

Beitz AJ, Ecklund LJ (1988) Colocalization of fixative-modified glutamate and glutaminase but not GAD in rubrospinal neurons. J Comp Neurol 274:265-279.

Bellocchio EE, Reimer RJ, Fremeau RT, Edwards RH (2000) Uptake of glutamate into synaptic vesicles by an inorganic phosphate transporter. Science 289:957-960.

Bianchi AL, Denavit-Saubie M, Champagnat J (1995) Central control of breathing in mammals: neuronal circuitry, membrane properties, and neurotransmitters. Physiol Rev 75:1-45.

Bourguignon JP, Gerard A, Alvarez Gonzalez ML, Purnelle G, Franchimont P (1995) Endogenous glutamate involvement in pulsatile secretion of gonadotropin-releasing hormone: evidence from effect of glutamine and developmental changes. Endocrinology 136:911-916.

Bradford HF, Ward HK, Foley P (1989) Glutaminase inhibition and the release of neurotransmitter glutamate from synaptosomes. Brain Res 476:29-34.

Butera Jr RJ, Rinzel J, Smith JC (1999) Models of respiratory rhythm generation in the pre-Botzinger complex. II. Populations of coupled pacemaker neurons. J Neurophysiol 82:398-415.

Chatziioannou A, Palaiologos G, Kolisis FN (2003) Metabolic flux analysis as a tool for the elucidation of the metabolism of neurotransmitter glutamate. Metab Eng 5:201-210.

Chaudhry FA, Reimer RJ, Edwards RH (2002) The glutamine commute: take the $\mathrm{N}$ line and transfer to the A. J Cell Biol 157:349-355.

Chen W, Mahadomrongkul V, Berger UV, Bassan M, DeSilva T, Tanaka K, Irwin N, Aoki C, Rosenberg PA (2004) The glutamate transporter GLT1a is expressed in excitatory axon terminals of mature hippocampal neurons. J Neurosci 24:1136-1148.

Conjard A, Brun V, Martin M, Baverel G, Ferrier B (2002) Effect of starvation on glutamine ammoniagenesis and gluconeogenesis in isolated mouse kidney tubules. Biochem J 368:301-308.

Conti F, Minelli A (1994) Glutamate immunoreactivity in rat cerebral cortex is reversibly abolished by 6-diazo-5-oxo-L-norleucine (DON), an inhibitor of phosphate-activated glutaminase. J Histochem Cytochem 42:717-726.

Curthoys NP (2001) Role of mitochondrial glutaminase in rat renal glutamine metabolism. J Nutr 131:2491S-2495S; discussion 2496S-2497S.

Curthoys NP, Lowry OH (1973) The distribution of glutaminase isoenzymes in the various structures of the nephron in normal, acidotic, and alkalotic rat kidney. J Biol Chem 248:162-168.

Curthoys NP, Watford M (1995) Regulation of glutaminase activity and glutamine metabolism. Annu Rev Nutr 15:133-159.

Danbolt NC (2001) Glutamate uptake. Prog Neurobiol 65:1-105.

Danbolt NC, Chaudhry FA, Dehnes Y, Lehre KP, Levy LM, Ullensvang K, Storm-Mathisen J (1998) Properties and localization of glutamate transporters. Prog Brain Res 116:23-43.

Del Negro CA, Johnson SM, Butera RJ, Smith JC (2001) Models of respiratory rhythm generation in the pre-Botzinger complex. III. Experimental tests of model predictions. J Neurophysiol 86:59-74.

Engel AK, Singer W (2001) Temporal binding and the neural correlates of sensory awareness. Trends Cogn Sci 5:16-25.

Farb C, Aoki C, Milner T, Kaneko T, LeDoux J (1992) Glutamate immunoreactive terminals in the lateral amygdaloid nucleus: a possible substrate for emotional memory. Brain Res 593:145-158.

Feldman JL, Smith JC (1989) Cellular mechanisms underlying modulation of breathing pattern in mammals. Ann NY Acad Sci 563:114-130.

Funk GD, Smith JC, Feldman JL (1993) Generation and transmission of respiratory oscillations in medullary slices: role of excitatory amino acids. J Neurophysiol 70:1497-1515.

Galfalvy HC, Erraji-Benchekroun L, Smyrniotopoulos P, Pavlidis P, Ellis SP, Mann JJ, Sibille E, Arango V (2003) Sex genes for genomic analysis in human brain: internal controls for comparison of probe level data extraction. BMC Bioinformatics 4:37.

Galloway MP, O’Leary-Moore SK, Seraji-Bozorgzad N, Moore HM, Miller GM, Rayport SG (2005) Probing the nature of MR-visible neurochemicals using transgenic mice lacking glutaminase and high-resolution magic 
angle spinning- ${ }^{1} \mathrm{H}$ magnetic resonance spectroscopy. Soc Neurosci Abstr 31:945.11.

Ghosh K, Van Duyne GD (2002) Cre-loxP biochemistry. Methods 28:374-383.

Gras C, Herzog E, Bellenchi GC, Bernard V, Ravassard P, Pohl M, Gasnier B, Giros B, El Mestikawy S (2002) A third vesicular glutamate transporter expressed by cholinergic and serotoninergic neurons. J Neurosci 22:5442-5451.

Haji A, Takeda R, Okazaki M (2000) Neuropharmacology of control of respiratory rhythm and pattern in mature mammals. Pharmacol Ther $86: 277-304$

Hertz L (2004) Intercellular metabolic compartmentation in the brain: past, present and future. Neurochem Int 45:285-296.

Hertz L, Dringen R, Schousboe A, Robinson SR (1999) Astrocytes: glutamate producers for neurons. J Neurosci Res 57:417-428.

Ishikawa T, Sahara Y, Takahashi T (2002) A single packet of transmitter does not saturate postsynaptic glutamate receptors. Neuron 34:613-621.

Jacquin TD, Borday V, Schneider-Maunoury S, Topilko P, Ghilini G, Kato F, Charnay P, Champagnat J (1996) Reorganization of pontine rhythmogenic neuronal networks in Krox-20 knockout mice. Neuron 17:747-758.

Jolimay N, Franck L, Langlois X, Hamon M, Darmon M (2000) Dominant role of the cytosolic C-terminal domain of the rat 5-HT1B receptor in axonal-apical targeting. J Neurosci 20:9111-9118.

Kaneko T, Mizuno N (1988) Immunohistochemical study of glutaminasecontaining neurons in the cerebral cortex and thalamus of the rat. J Comp Neurol 267:590-602.

Kvamme E, Roberg B, Torgner IA (2000) Phosphate-activated glutaminase and mitochondrial glutamine transport in the brain. Neurochem Res 25:1407-1419.

Laake JH, Takumi Y, Eidet J, Torgner IA, Roberg B, Kvamme E, Ottersen OP (1999) Postembedding immunogold labelling reveals subcellular localization and pathway-specific enrichment of phosphate activated glutaminase in rat cerebellum. Neuroscience 88:1137-1151.

Lakso M, Sauer B, Mosinger Jr B, Lee EJ, Manning RW, Yu SH, Mulder KL, Westphal H (1992) Targeted oncogene activation by site-specific recombination in transgenic mice. Proc Natl Acad Sci USA 89:6232-6236.

Mulkey DK, Stornetta RL, Weston MC, Simmons JR, Parker A, Bayliss DA, Guyenet PG (2004) Respiratory control by ventral surface chemoreceptor neurons in rats. Nat Neurosci 7:1360-1369.

Ottersen OP, Zhang N, Walberg F (1992) Metabolic compartmentation of glutamate and glutamine: morphological evidence obtained by quantitative immunocytochemistry in rat cerebellum. Neuroscience 46:519-534.

Papes F, Surpili MJ, Langone F, Trigo JR, Arruda P (2001) The essential amino acid lysine acts as precursor of glutamate in the mammalian central nervous system. FEBS Lett 488:34-38.

Peng L, Hertz L, Huang R, Sonnewald U, Petersen SB, Westergaard N, Larsson O, Schousboe A (1993) Utilization of glutamine and of TCA cycle constituents as precursors for transmitter glutamate and GABA. Dev Neurosci 15:367-377.

Perrais D, Ropert N (1999) Effect of zolpidem on miniature IPSCs and occupancy of postsynaptic $\mathrm{GABA}_{\mathrm{A}}$ receptors in central synapses. J Neurosci 19:578-588.

Pin JP, Weiss S, Sebben M, Kemp DE, Bockaert J (1986) Release of endog- enous amino acids from striatal neurons in primary culture. J Neurochem 47:594-603.

Polan HJ, Eljuga L (2002) Involvement of maternally-directed orienting behaviors in the oral nipple grasp from birth to day 2. Dev Psychobiol 41:320.

Polan HJ, Milano D, Eljuga L, Hofer MA (2002) Development of rats' maternally directed orienting behaviors from birth to day 2. Dev Psychobiol 40:81-103.

Ramirez JM, Quellmalz UJ, Richter DW (1996) Postnatal changes in the mammalian respiratory network as revealed by the transverse brainstem slice of mice. J Physiol (Lond) 491:799-812.

Ritter B, Zhang W (2000) Early postnatal maturation of $\mathrm{GABA}_{\mathrm{A}}$-mediated inhibition in the brainstem respiratory rhythm-generating network of the mouse. Eur J Neurosci 12:2975-2984.

Sauvinet V, Parrot S, Benturquia N, Bravo-Moraton E, Renaud B, Denoroy L (2003) In vivo simultaneous monitoring of gamma-aminobutyric acid, glutamate, and L-aspartate using brain microdialysis and capillary electrophoresis with laser-induced fluorescence detection: analytical developments and in vitro/in vivo validations. Electrophoresis 24:3187-3196.

Smith JC, Ellenberger HH, Ballanyi K, Richter DW, Feldman JL (1991) PreBotzinger complex: a brainstem region that may generate respiratory rhythm in mammals. Science 254:726-729.

Srinivas S, Watanabe T, Lin CS, William CM, Tanabe Y, Jessell TM, Costantini F (2001) Cre reporter strains produced by targeted insertion of EYFP and ECFP into the ROSA26 locus. BMC Dev Biol 1:4.

Stern JM (1997) Offspring-induced nurturance: animal-human parallels. Dev Psychobiol 31:19-37.

Sulzer D, Joyce MP, Lin L, Geldwert D, Haber SN, Hattori T, Rayport S (1998) Dopamine neurons make glutamatergic synapses in vitro. J Neurosci 18:4588-4602.

Sweatt AJ, Garcia-Espinosa MA, Wallin R, Hutson SM (2004) Branchedchain amino acids and neurotransmitter metabolism: expression of cytosolic branched-chain aminotransferase (BCATc) in the cerebellum and hippocampus. J Comp Neurol 477:360-370.

Thoby-Brisson M, Ramirez JM (2000) Role of inspiratory pacemaker neurons in mediating the hypoxic response of the respiratory network in vitro. J Neurosci 20:5858-5866.

Thoby-Brisson M, Cauli B, Champagnat J, Fortin G, Katz DM (2003) Expression of functional tyrosine kinase $B$ receptors by rhythmically active respiratory neurons in the pre-Botzinger complex of neonatal mice. J Neurosci 23:7685-7689.

Varoqui H, Zhu H, Yao D, Ming H, Erickson JD (2000) Cloning and functional identification of a neuronal glutamine transporter. J Biol Chem 275:4049-4054.

Waagepetersen HS, Qu H, Sonnewald U, Shimamoto K, Schousboe A (2005) Role of glutamine and neuronal glutamate uptake in glutamate homeostasis and synthesis during vesicular release in cultured glutamatergic neurons. Neurochem Int 47:92-102.

Weston MC, Stornetta RL, Guyenet PG (2004) Glutamatergic neuronal projections from the marginal layer of the rostral ventral medulla to the respiratory centers in rats. J Comp Neurol 473:73-85.

Yudkoff M, Daikhin Y, Nissim I, Horyn O, Luhovyy B, Lazarow A, Nissim I (2005) Brain amino acid requirements and toxicity: the example of leucine. J Nutr 135:1531S-1538S. 